\title{
The technique of robotic anatomic pulmonary segmentectomy I: right sided segments
}

\author{
Farid Gharagozloo, Mark Meyer \\ Center for Advanced Thoracic Surgery, Global Robotics Institute, Advent Health Celebration, University of Central Florida, \\ Celebration, FL 34786, USA.
}

Correspondence to: Dr. Farid Gharagozloo, Center for Advanced Thoracic Surgery, Global Robotics Institute, Advent Health Celebration, University of Central Florida, 400 Celebration Place, Celebration, FL 34786, USA.

E-mail: farid.gharagozloo.md@adventhealth.com

How to cite this article: Gharagozloo F, Meyer M. The technique of robotic anatomic pulmonary segmentectomy I: right sided segments. Mini-invasive Surg 2020;4:66. http://dx.doi.org/10.20517/2574-1225.2020.53

Received: 27 May 2020 First Decision: 13 Jul 2020 Revised: 13 Jul 2020 Accepted: 20 Jul 2020 Published: 12 Oct 2020

Academic Editor: Giulio Belli Copy Editor: Cai-Hong Wang Production Editor: Jing Yu

\begin{abstract}
Anatomic pulmonary segmentectomy and mediastinal nodal dissection have been advocated in patients with smaller tumors or patients with limited pulmonary reserve. The overall five-year survival and lung cancer-specific five-year survival following anatomic segmentectomy have been shown to be equivalent to lobectomy. Robotic surgical systems have the advantage of magnified high-definition three-dimensional visualization and greater instrument maneuverability in a minimally invasive platform. Robotics can facilitate the dissection of the bronchovascular structures and replicate the technique of segmentectomy by thoracotomy. Greater experience with the robotic platform has resulted in a reproducible technique. The Technique of Robotic Anatomic Segmentectomy Part I outlines a stepwise approach to robotic segmentectomy of S1, S2, S3, S4, S5, S6, and S7-S10 of the right lung. The Technique of Robotic Anatomic Segmentectomy Part II outlines a stepwise approach to robotic segmentectomy to the left lung.
\end{abstract}

Keywords: Robotic, segmentectomy, lung cancer, superior segment, anterior segment, apicoposterior segment, basal segment, sublobar resection

\section{INTRODUCTION}

Anatomic segmental resection (segmentectomy) is the excision of one or more bronchopulmonary segments of a pulmonary lobe with individual ligation and division of the corresponding broncho-vascular

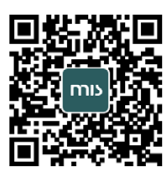


structures. It is important to differentiate segmentectomy from "wedge resection", which is a form of sublobar resection. A wedge resection is defined as removal of a portion of the lung along non-anatomic planes usually with the aid of a stapling device.

Controversy about sublobar lung resection is largely attributed to The Lung Cancer Study Group's prospectively randomized study in 1995 which showed that sublobar resections (a combined cohort of anatomic segmentectomies and wedge resections) had 75\% increased recurrence, 30\% increased overall death, and 50\% increased cancer-related death compared to lobectomy ${ }^{[1,2]}$. This study did not differentiate between anatomic segmentectomy and wedge resection. Subsequently, multiple retrospective studies have shown that in general segmentectomies have lower recurrence rates and better survival than wedge resections ${ }^{[3-7]}$. On the other hand, in a retrospective study of patients with T1No disease, Altorki et al. ${ }^{[8]}$ showed that anatomic segmentectomy and wedge resection are comparable oncologic procedures. In addition, although anatomic segmentectomy was associated with a more thorough lymph node dissection, it did not offer a survival advantage in this group of patients with early disease. Although prospective studies comparing wedge resection and anatomic segmentectomy for T1No disease are in progress, anatomic segmentectomy may be a better oncologic procedure in patients with more advanced disease. Furthermore, multiple retrospective studies have demonstrated no significant difference in oncologic outcomes with anatomic segmentectomy versus lobectomy ${ }^{[9-12]}$.

As the result of these findings, recently, there has been renewed interest in segmentectomy for small primary lung cancer tumors, as well as in patients with marginal pulmonary reserve $e^{[13]}$.

Robotic surgical systems have the advantage of high definition three-dimensional visualization, precise instrument maneuverability in a confined space, and decreased surgeon fatigue. The surgical robot is ideally suited for performing minimally invasive anatomic segmentectomy. It allows for precise dissection of the segmental bronchopulmonary structures while minimizing trauma to surrounding tissue and allows for thorough complete dissection of the mediastinal nodes. Dylewski reported the first experience with robotic anatomic segmentectomy ${ }^{[14]}$. In this study, robotic segmentectomy had a lower complication rate than robotic lobectomy (11.4\% vs. 31\%). Pardolesi and Cerfolio reported a similar experience ${ }^{[15-17]}$. Demir reported that VATS and robotic segmentectomy have similar morbidity and mortality ${ }^{[18]}$. Nguyen et al. ${ }^{[19]}$ used the Standard da Vinci and Si da Vinci platforms and replicated the anatomic segmentectomy technique as performed by a thoracotomy. In their experience, the robotic approach was used for the apical, anterior, and posterior segments of the upper lobe; the superior segment of the lower lobe on the right; the apical, anterior, and posterior segments of the upper lobe; and the lingual and superior segment of the lower lobe on the left.

This paper outlines a step by step approach to robotic anatomic segmentectomy to the segments of the right lung. The segmental anatomy of the right lung is illustrated in Figure 1.

\section{RIGHT SIDED SEGMENTECTOMY}

\section{Port placement}

Port placement for anatomic segmentectomy is the same as with pulmonary lobectomy.

The operating room table is reversed such that the pedestal does not interfere with the docking of the robot over the head of the patient.

A double lumen endotracheal tube is placed, and the patient is positioned in a full lateral decubitus position. The double lumen endotracheal tube is preferable to a bronchial blocker. The manipulation of the lung and the hilum can dislodge the bronchial blocker and result in loss of exposure during robotic 


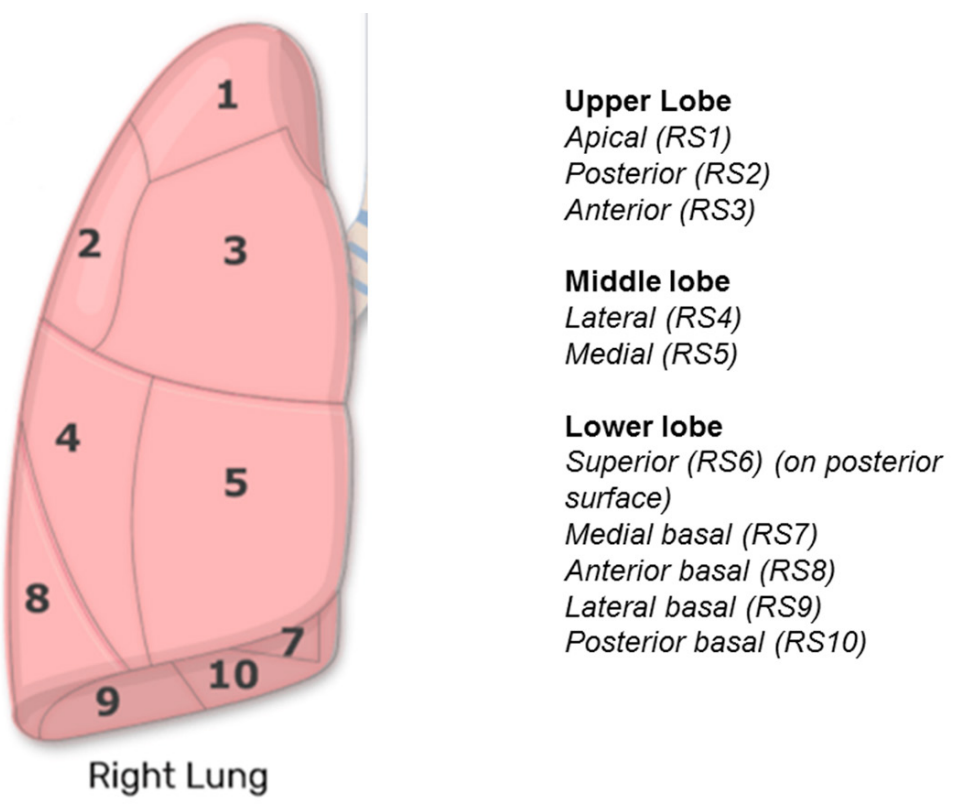

Figure 1. Right lung bronchopulmonary segments

lung surgery. In addition, as the robot is positioned over the head of the patient, with the robot in place, manipulation and replacement of the endobronchial blocker is very cumbersome. The right arm is placed over pillows and positioned high enough such that access to the 4th intercostal space in the anterior axillary line is readily attained. The table is flexed to move the hip down and to open the intercostal spaces. The lung is deflated and placed on suction. The position of the double lumen tube is rechecked after the patient is prepped and draped. It is imperative that the lung remain isolated throughout the procedure.

Figures 2 and 3 show the right chest port placement. A line is drawn from the tip of the scapula to the costal arch. This delineates the highest point in the chest and the midscapular line (corresponding to the posterior axillary line with the arm at the side of the patient). Pleural entry is with a Hassan needle. Saline is infused and care is taken to look for easy egress of the saline from the needle. If there is any question of pleural adhesions, the Visiport Instrument (Medtronic Inc. Norwalk, Conn, USA) is used for entry into the pleural space under direct vision. If the Visiport is used, a purse string is placed in the muscle layer and tied around the robot camera port to prevent $\mathrm{CO}_{2}$ leakage.

Port \#1 is the camera port. Warm, humidified $\mathrm{CO}_{2}$ is insufflated through this port at a flow of $6 \mathrm{~L} / \mathrm{min}$ to a pressure of 6-8 $\mathrm{mmHg}$ in order to push the lung and diaphragm away. The other ports are placed under direct vision. Port \#2 $(8 \mathrm{~mm})$ is placed in the 7th intercostal space in the poster scapular line. This port is $9 \mathrm{~cm}$ posterior to Port \#1 and accommodates da Vinci arm \#2. Prior to the placement of Port \#3, a 21-gauge needle is inserted into the 7th intercostal space at costovertebral junction from the patient's back and injects a $10-\mathrm{mL}$ subpleural bubble of $0.25 \%$ bupivacaine with epinephrine near the intercostal nerve. Next, Port \#3 is placed $10 \mathrm{~cm}$ posterior to Port \#2 in the 7th intercostal space just medial to the spine. This port accommodates da Vinci arm \#3. Port \#4 is placed $9 \mathrm{~cm}$ anterior to Port \# 1 in the 7 th intercostal space at the anterior scapular line. This port accommodates da Vinci arm \#1. The Assistant Port \#5 uses a 10-12 Versiport (Medtronic Inc. Norwalk, Conn, USA) trocar, is placed in the 9th intercostal space, and is triangulated between Ports \#1 and \#4. It should be two or three ribs lower than the da Vinci ports and as far away as possible to maximize assistant workspace. Keeping this port off the trajectory lines for the other ports will facilitate the patient-side assistant's access for retraction and other maneuvers. In total, including the vitally important assistant port, robotic anatomic segmentectomy is performed with five ports. The use of 


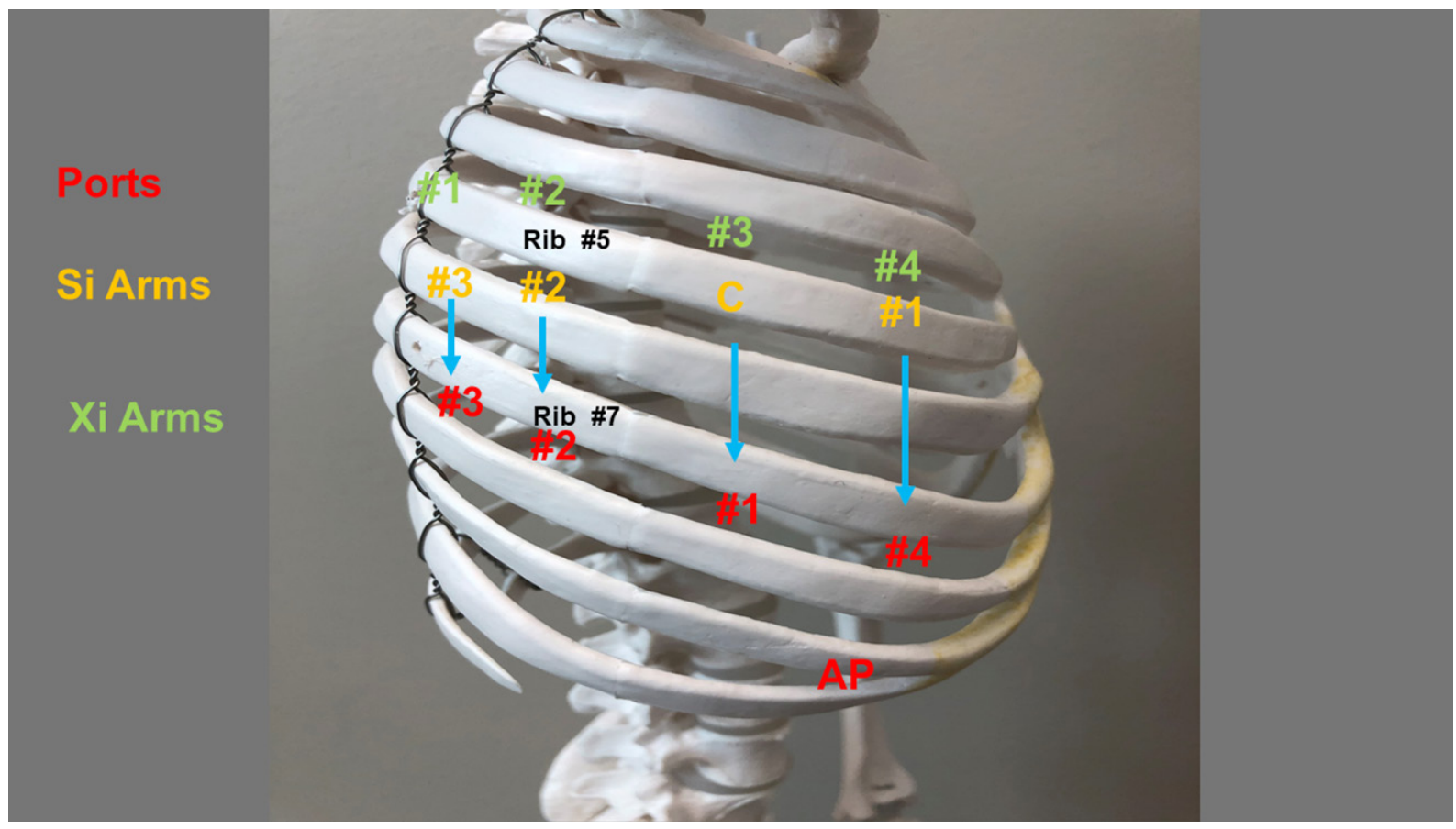

Figure 2. Schematic of port placement for robotic segmentectomy in the right chest. Ports are in red, $\mathrm{Si}$ arms are in yellow, and $\mathrm{Xi}$ arms are in green. AP: assistant port

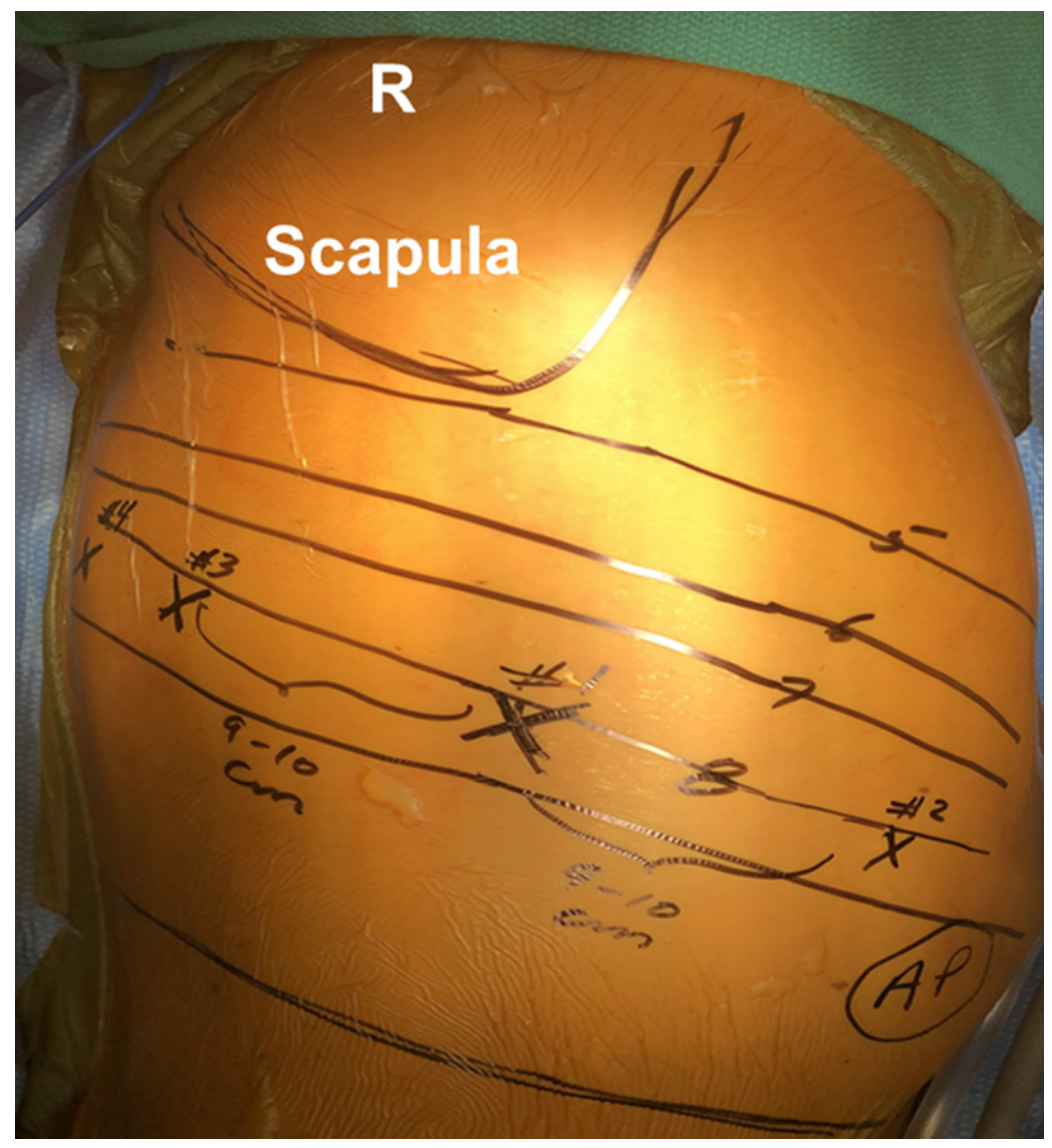

Figure 3. Port placement for robotic segmentectomy in the right chest. Patient is in full lateral decubitus position. Robot arms are numbered $\# 1, \# 2, \# 3$, and \#4. AP: assistant port 
additional ports should be tailored to the specific situation and the experience of the surgeon. Surgeons are encouraged to use as many ports as are necessary to perform a safe and oncologically efficacious anatomic segmentectomy.

Port placement and intercostal sites are the same for all segments. All effort should be made to keep the distance between the ports as close as possible as to what is described above. In smaller patients, care must be taken to keep the trocar sites as far as possible and within the parameters that are outlined. This strategy prevents interference in arm function with the present robotic platforms. It is possible that port placement may be modified in the future with the development of new platforms and robot arms which may have a smaller "footprint" on the chest.

Port Placement with Si Robot: Robotic arm \#3 is located two $\mathrm{cm}$ lateral from the spinous process of the vertebral body, robotic arm $\# 2$ is $10 \mathrm{~cm}$ medial to robotic arm \#3, the camera port (we prefer the $12-\mathrm{mm}$ camera) is $9 \mathrm{~cm}$ medial to robotic arm \#2, and robotic arm \#1 is placed right above the diaphragm anteriorly.

Port Placement with Xi Robot: For the Xi system, the ports are placed in slightly different locations. They are also numbered differently. Robotic arm \#1 is placed $4 \mathrm{~cm}$ away from the spinous process. Robotic arm \#2 is placed $8 \mathrm{~cm}$ from arm \#1 and robotic arm \#3 is placed $8 \mathrm{~cm}$ from arm \#2. Robotic arm \#4 is placed right above the diaphragm anteriorly. The assistant port is triangulated behind the camera arm and robotic arm \#4 in a similar fashion. The camera is carried by arm \#3. Arms \#1-\#4 are all placed in the 7th intercostal space. The Xi robot has the advantage of providing the robotic stapler, which gives the surgeon control of the stapling and the use of indocyanine green dye for identification of the intersegmental plane.

Instruments: $0^{\circ}$ and $/$ or $30^{\circ}$ down viewing endoscope, $5 \mathrm{~mm}$ Thoracic Grasper (left (3), Cadiere Forceps (left (2)), and Curved Bipolar Dissector (right (1)).

\section{Mediastinal nodal dissection}

Complete nodal dissection is performed with all anatomic segmentectomy procedures. Begin by dividing the inferior pulmonary ligament and remove Station \#9 and \#8 nodes [Figure 4]. The most posterior arm is used to retract the lower lobe medially and anteriorly to remove lymph nodes from Station \#7. Next, open the pleura anterior to the vagus nerve and divide the anterior branch of the nerve which traverses the subcarinal space. At the beginning of the case, a nasogastric tube should be inserted to decompress the stomach. After decompression of the stomach, some surgeons may prefer to remove the nasogastric tube to aid in the retraction of the esophagus during the subcarinal dissection. This opens the subcarinal space and allows for better access to the Station \#7 nodes. Identify the right mainstem bronchus and stay posterior to the edge of the cartilage. Remove the Station \#7 nodes and control the subcarinal artery at the carina. At the end of the dissection, the right and left mainstem bronchi should be visible and the posterior aspect of the pericardium should be cleaned and clearly visible [Figure 5]. Next, the most posterior arm is used to retract the upper lobe inferiorly during dissection of Stations $\# 2 \mathrm{R}$ and $\# 4 \mathrm{R}$, clearing the space between the superior vena cava anteriorly, the trachea posteriorly, and the azygos vein inferiorly [Figure 6].

Completion of the lymph node dissection opens the mediastinal space and facilitates the dissection of the artery and the bronchus. After identifying the right mainstem bronchus, it is followed up to the level of Station \#10R lymph node [Figure 7]. This node is superior to the right mainstem bronchus. It should be dissected and swept towards the lung, thereby exposing the bronchus and the truncus branch of the pulmonary artery.

Dissection is continued and the crotch between the right upper lobe bronchus and bronchus intermedius is defined. All Station \#7 and \#11 nodes and the sump node are removed. This maneuver facilitates later 


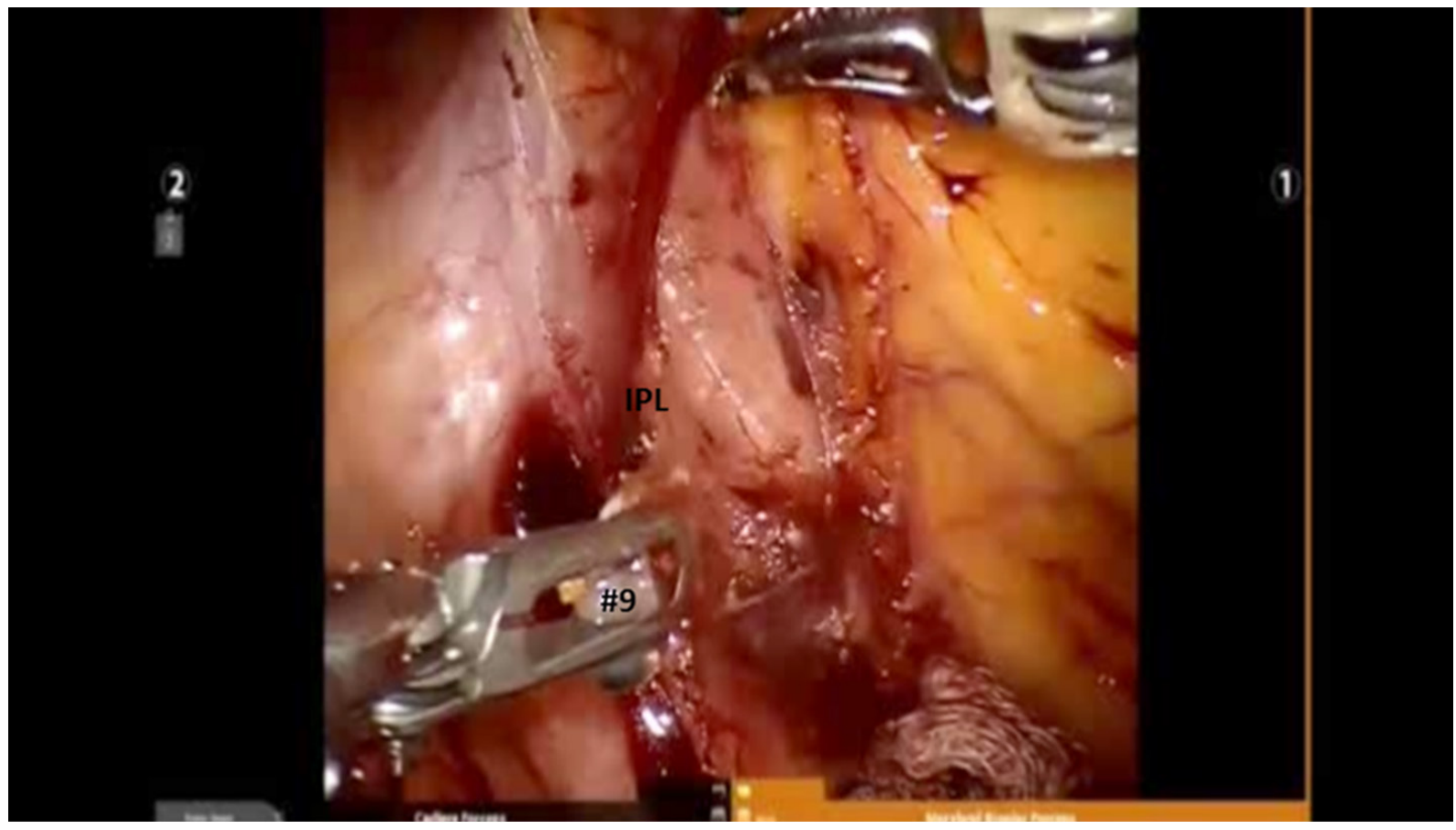

Figure 4. The IPL is divided and Station \# 9 nodes are removed. IPL: inferior pulmonary ligament

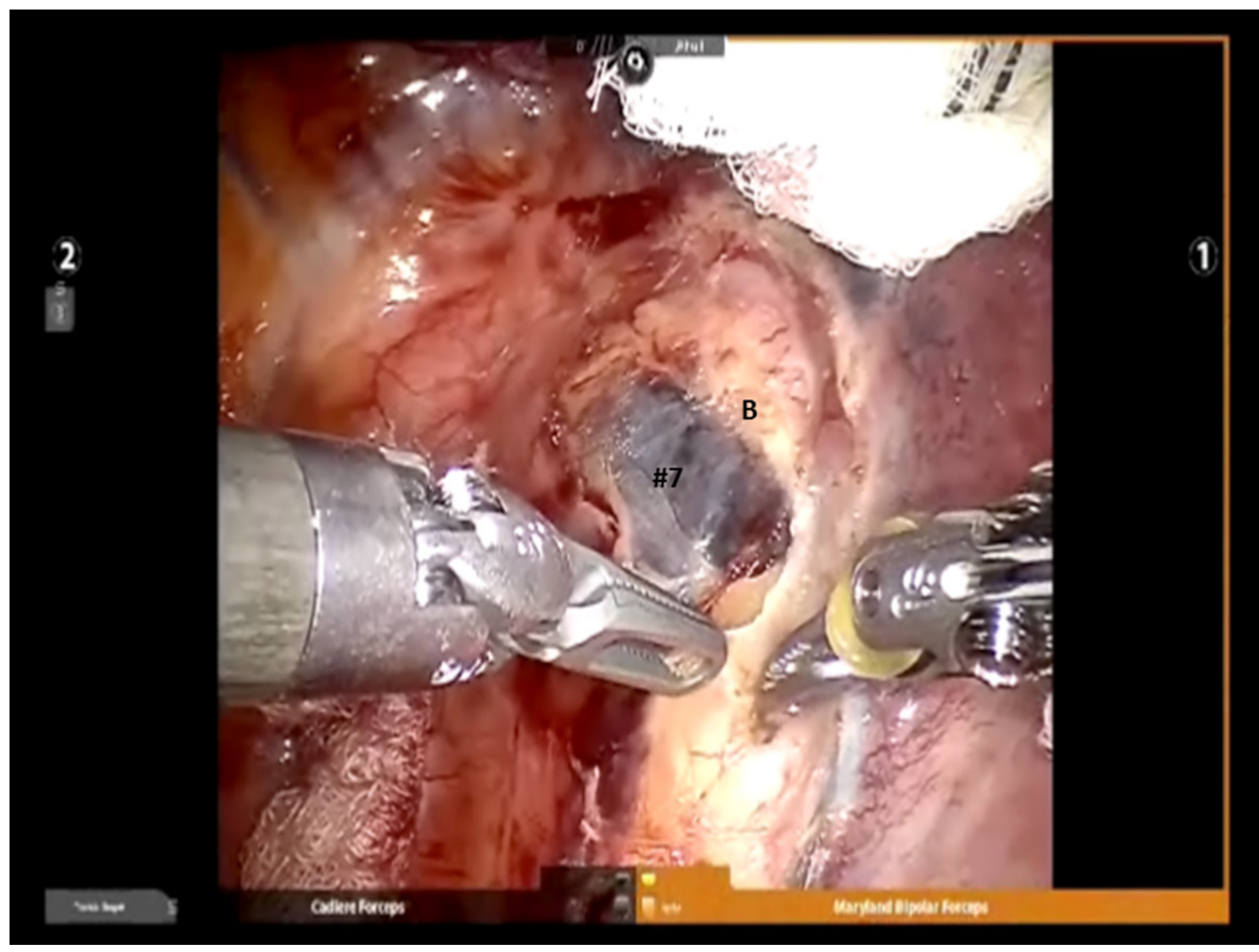

Figure 5. Subcarinal dissection: View of the right mainstem bronchus (B) and Station \#7 lymph nodes 


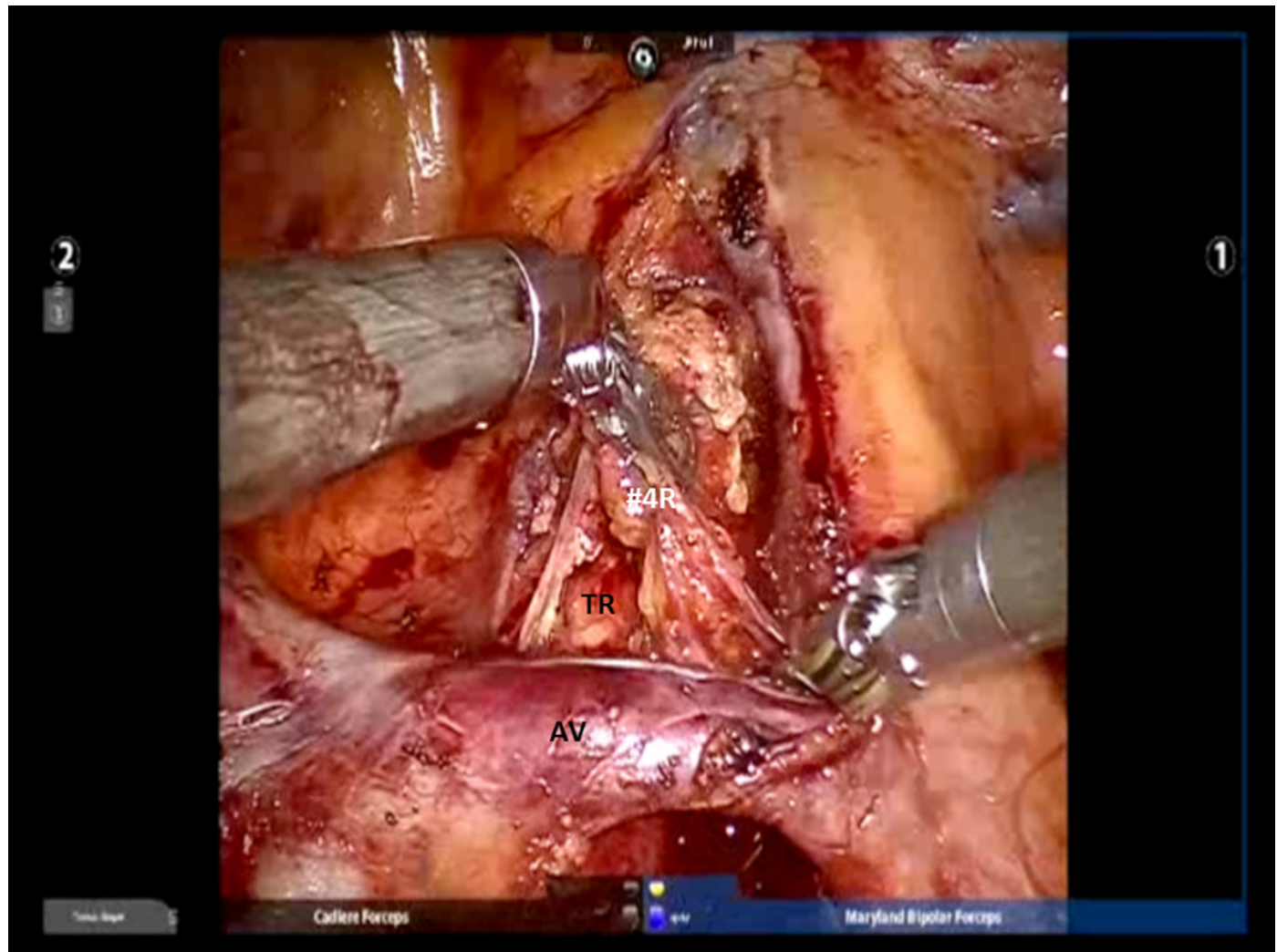

Figure 6. Dissection of the right paratracheal space: View of the \#4R nodal bundle, AV, and TR. AV: azygous vein; TR: trachea

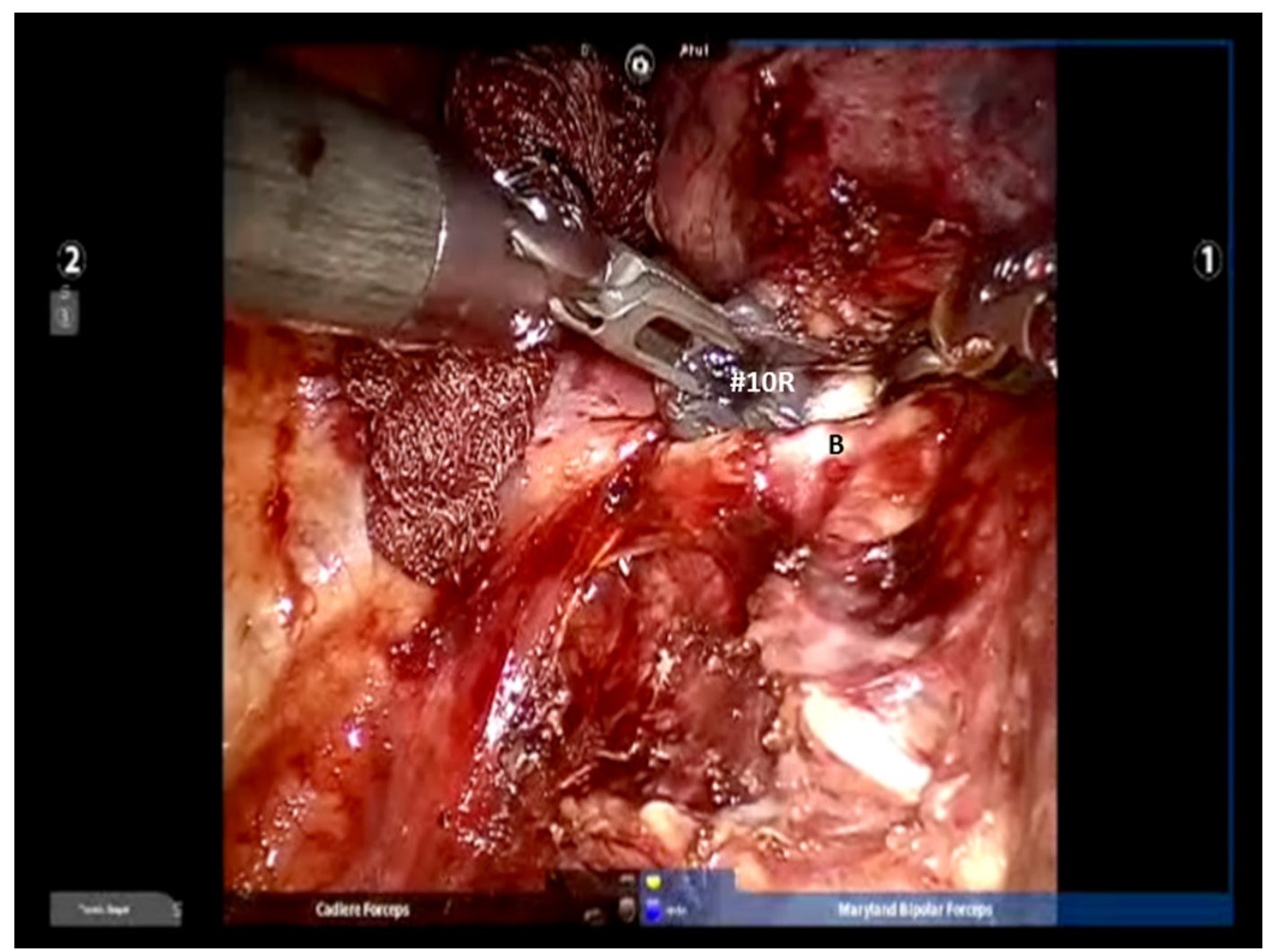

Figure 7. Dissection of \#10R Nodal bundle: The nodes are above the right mainstem bronchus (B). Removal of the nodes facilitates getting around the bronchus 




Figure 8. RS1 segmentectomy: Dissection of the $A 1$ branch of the PA. View of the $S V C, A Z$, and the right main PA. AZ: azygous vein; PA: pulmonary artery; SVC: superior vena cava

dissection of the pulmonary artery (PA) as well as the right upper lobe bronchus. In addition, the removal of Station \#11 nodes facilitates the completion of the posterior fissure.

Next, the lung is retracted posteriorly to expose the anterior hilum. The dissection is carried down between the hilar structures and the phrenic nerve. The phrenic nerve is swept down to remove the \#10R lymph node. The bifurcation between the middle and upper lobe veins is dissected. It is best to encircle the entire upper lobe vein off the underlying PA using the Cadiere Forceps in the left arm and pass a red rubber vessel loop to elevate the vein. This makes the dissection of the middle lobe vein easier. Following the dissection of the middle lobe vein, the Cadiere Forceps is passed under the elevated upper lobe vein, and the vessel loop is released and regrasped, thereby isolating the upper lobe vein. Dissection is continued and the proximal main PA is exposed as it emerges from the pericardium.

\section{Right upper lobe anatomic apical segmentectomy (S1)}

Following the complete mediastinal nodal dissection, the lung is retracted in a caudal direction and the A1 PA branch is identified, dissected away from the descending branch of the right PA, and divided using a stapler with a vascular cartridge [Figures 8 and 9]. Next, the lung is retracted posteriorly and the V1 branch of the superior pulmonary vein is identified [Figure 9]. The N1 nodal bundle which resides between the V1 and the PA is removed. V1 is encircled and divided with a stapler with a vascular cartridge. Although many surgeons do not anatomically isolate and divide the segmental vein separately, isolation and division of the vein helps in opening the operative space and may be preferred. Division of the vessels and removal of the 


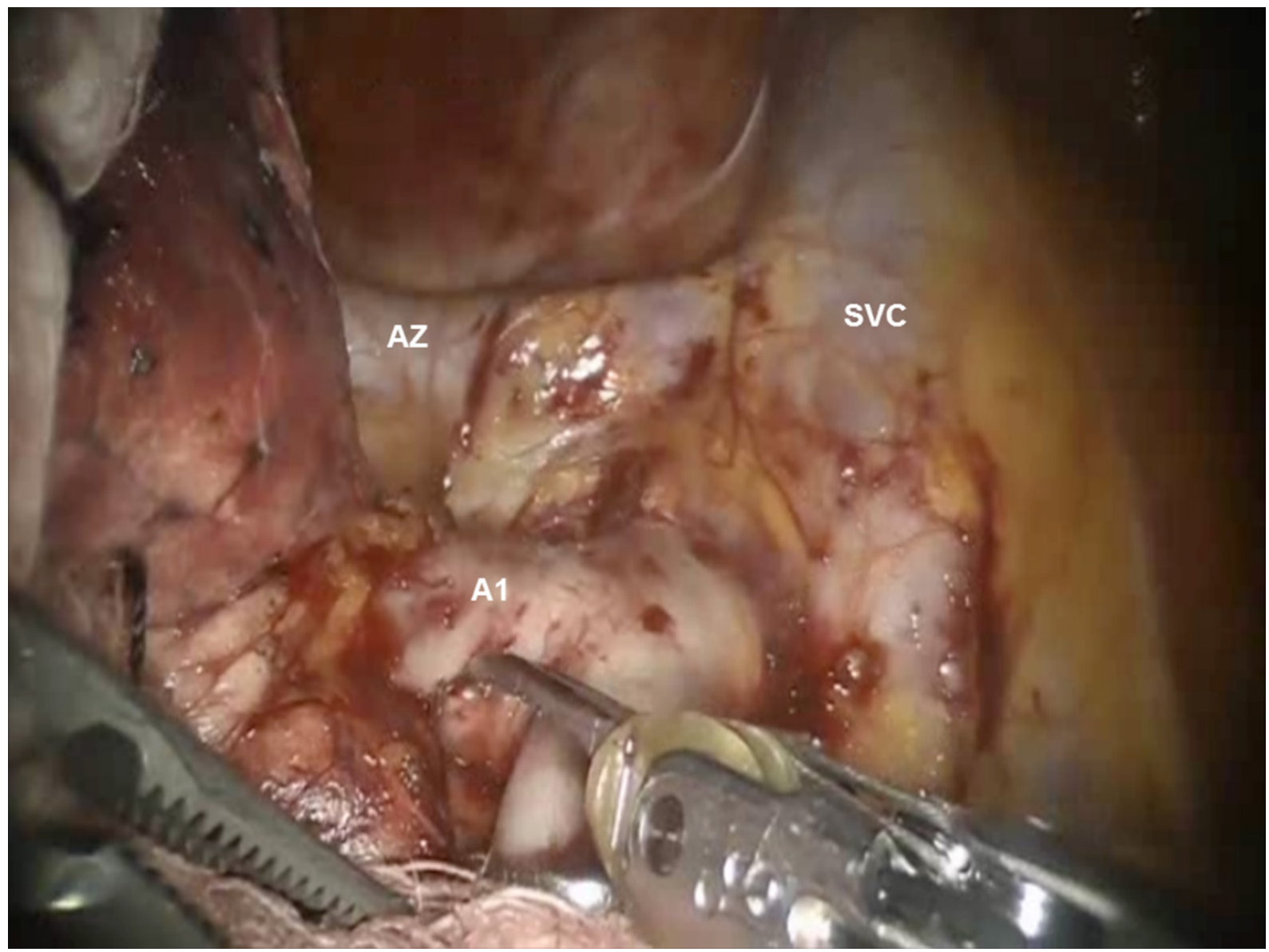

Figure 9. RS1 segmentectomy: Dissection of the A1 branch of the pulmonary artery. View of the SVC, $A Z$, and the right main pulmonary artery. AZ: azygous vein; SVC: superior vena cava

$\mathrm{N} 1$ nodes between the PA and the bronchus exposes the B1 bronchus. The B1 bronchus is encircled and divided with a stapler using a purple cartridge [Figure 10]. Prior to firing the stapler, the anesthesiologist must make certain that the suction catheter in the endotracheal tube is removed. Following the division of the $\mathrm{B} 1$ bronchus, if using the $\mathrm{Xi}$ robot, indocyanine green is injected and the fissure between the $\mathrm{S}_{1}$ and $\mathrm{S}_{3}$ segments is clearly delineated [Figure 11]. If using the Si robot, the anesthesiologist is instructed to gently inflate the lung to delineate the intersegmental fissure between S1 and S3. The intersegmental fissure is divided using a stapling device.

For all anatomic segmentectomies, the specimen retrieval bag (Anchor bag) is introduced through the accessory port; the specimen is placed into the bag; and the bag is removed.

\section{Right upper lobe anatomic posterior segmentectomy (S2)}

Following the complete mediastinal nodal dissection, the PA is identified in the oblique fissure [Figure 12]. Dissection is carried over the descending branch of the PA under the posterior aspect of the oblique fissure and cephalad to the superior segmental PA. The posterior oblique fissure is encircled with a vessel loop, retracted away from the PA, and divided with a stapling device. The upper lobe is retracted antero-medially, and dissection is carried cephalad on the PA until the truncus branches are identified. The V2 vein runs posteriorly from the superior pulmonary vein in the oblique fissure [Figure 13]. The V2 vein is dissected, encircled, and divided. Division of the V2 vein uncovers the A2 PA branch [Figure 14]. This is encircled with a vessel loop and divided using a stapler with a vascular cartridge. Next, the B2 bronchus is identified, 


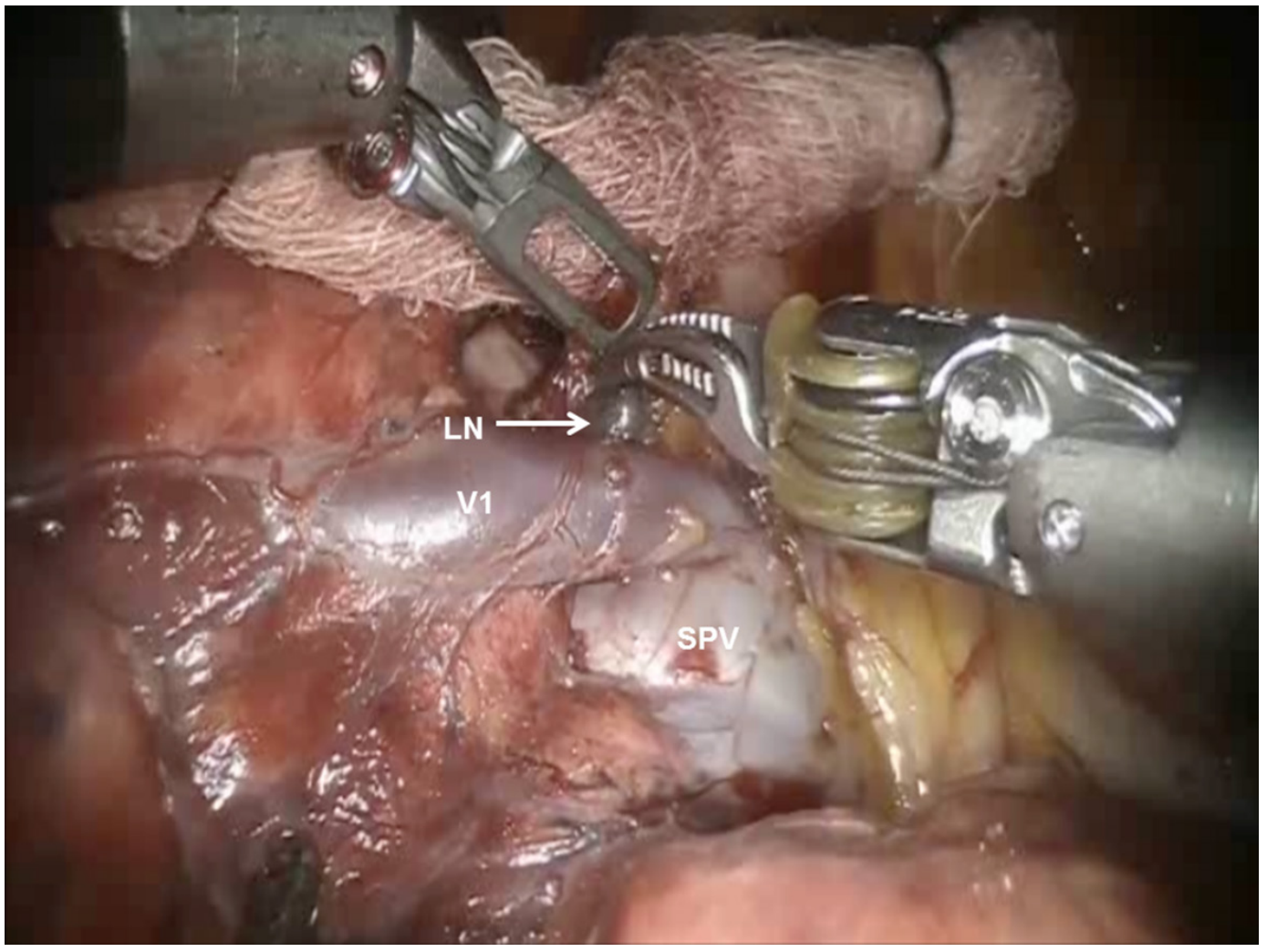

Figure 10. RS1 segmentectomy: The lung is retracted posteriorly and the V1 branch of the superior pulmonary vein is identified. View of the V1 pulmonary vein branch and SPV. LN: lymph node; SPV: superior pulmonary vein

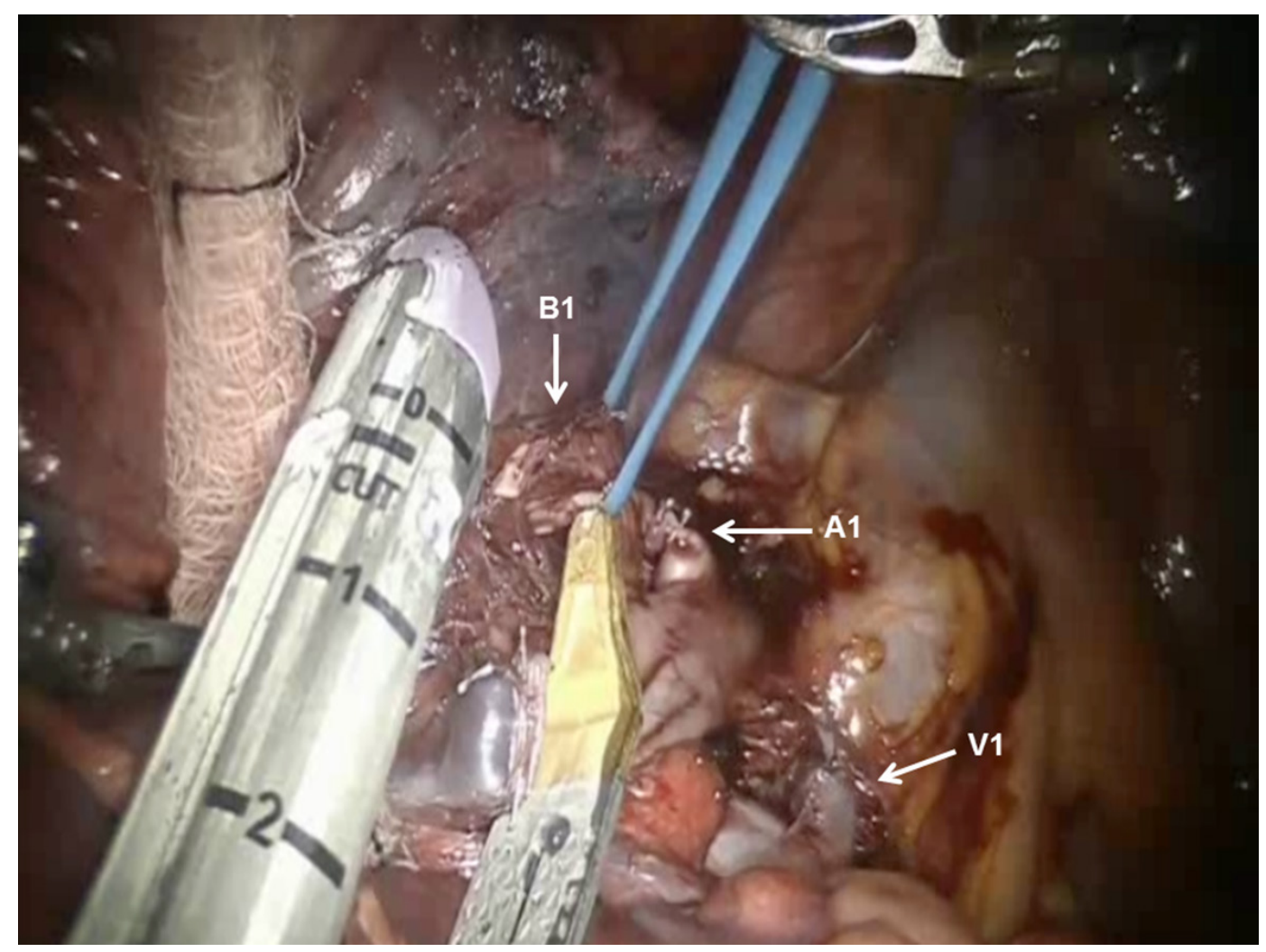

Figure 11. RS1 segmentectomy: B1 bronchus is encircled and divided with a stapler using a purple cartridge. Stapled stumps of the A1 Pulmonary artery and V1 Pulmonary vein branch are seen 


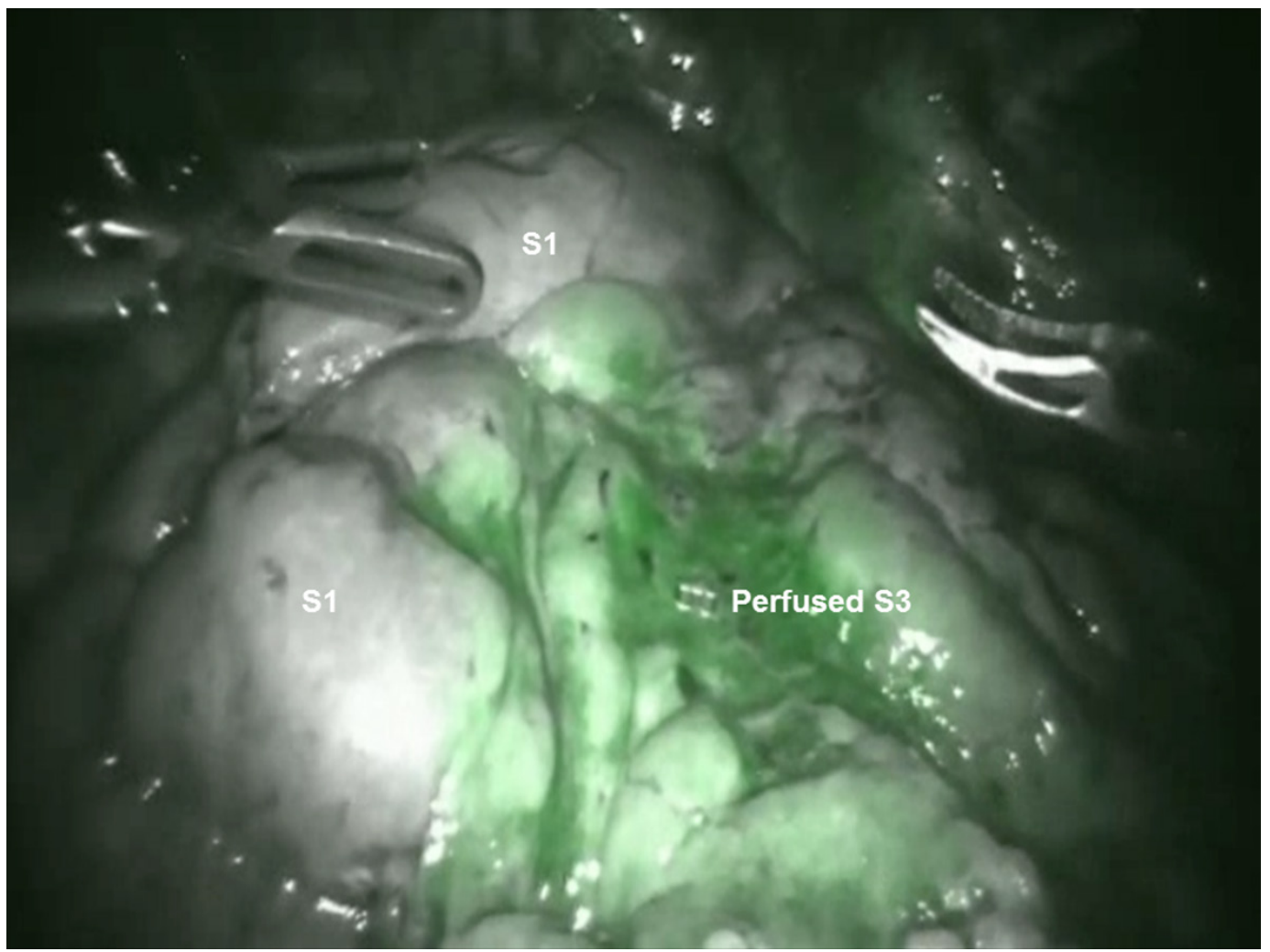

Figure 12. RS1 segmentectomy: Indocyanine green is injected and the fissure between the S1 and S3 segments is clearly delineated

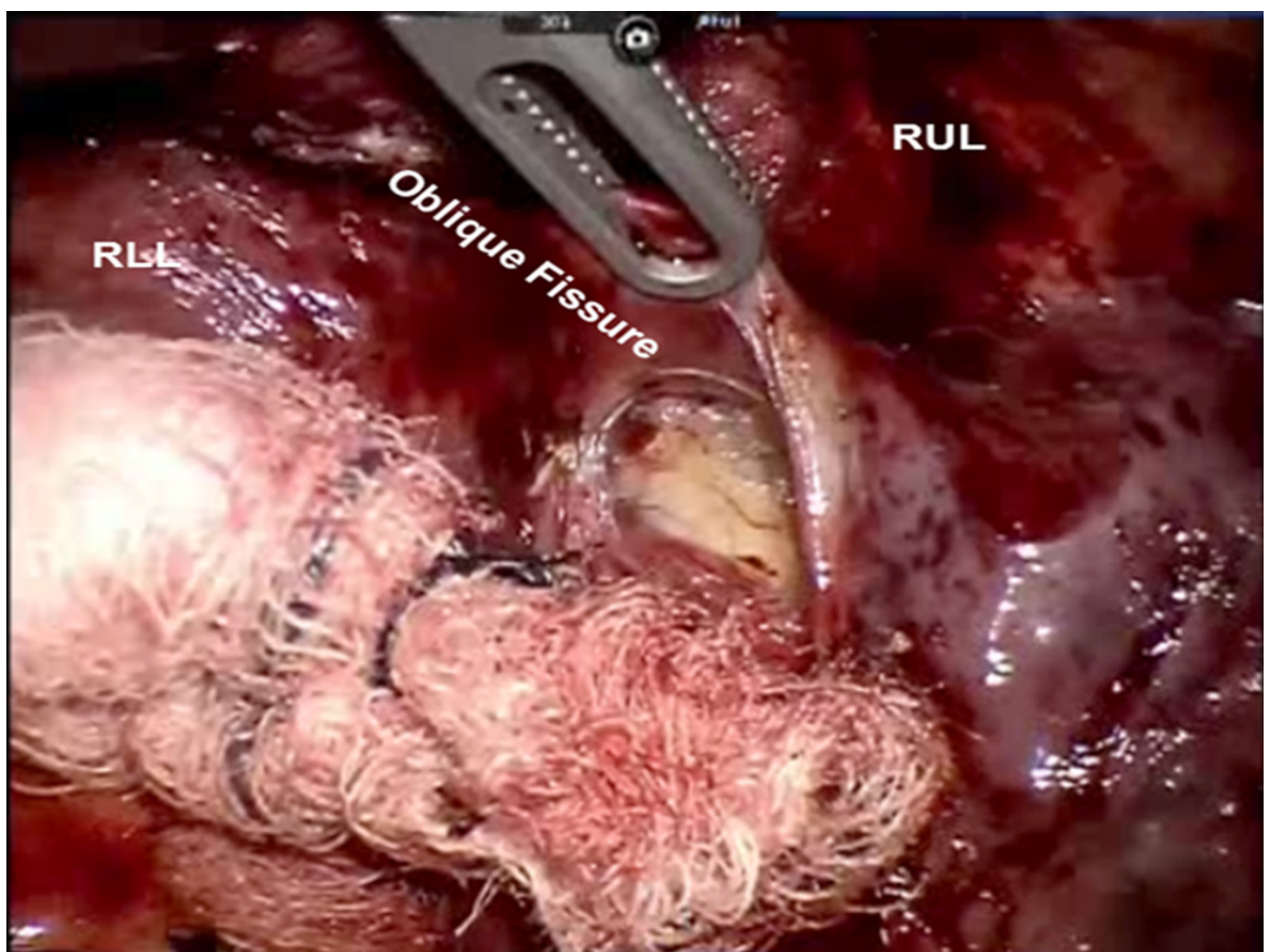

Figure 13. RS2 segmentectomy: Dissection is carried over the descending branch of the pulmonary artery under the posterior aspect of the oblique fissure and cephalad to the superior segmental pulmonary artery. RLL: right lower lobe; RUL: right upper lobe 


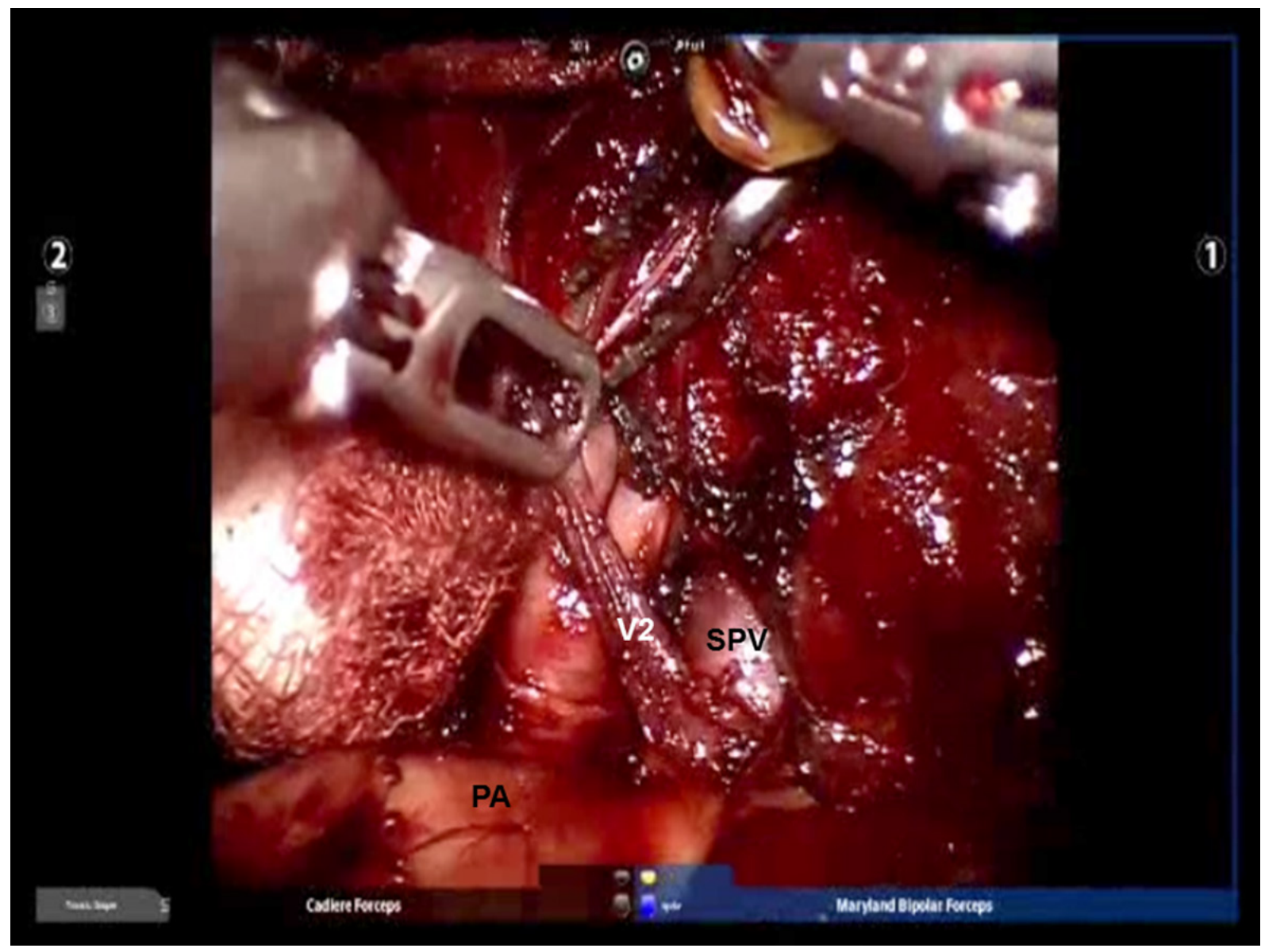

Figure 14. RS2 segmentectomy: The V2 vein runs posteriorly from the SPV in the oblique fissure. PA: pulmonary artery; SPV: superior pulmonary vein

the peribronchial $\mathrm{N}_{1}$ nodes are removed completely, the B2 bronchus is encircled with a vessel loop and divided with a stapler [Figure 15]. Finally, the intersegmental fissure is identified as described above and divided with a stapling device [Figure 16].

\section{Right upper lobe anatomic anterior segmentectomy (S3)}

Following the complete mediastinal nodal dissection and identification of the superior pulmonary vein, the descending trunk of the PA is approached in the oblique fissure. The nodes overlying the PA are removed. Dissection is carried cephalad in the sub adventitial plane, which overlies the PA toward the main trunk heading toward the space between the PA and superior pulmonary vein [Figure 17]. This maneuver elevates the transverse fissure of the lung away from the descending branch of the PA. A guide catheter is passed from a posterior to anterior direction under the transverse fissure, followed by a stapling device with a purple cartridge in the same direction, and the transverse fissure is divided. Division of the transverse fissure exposes the V3 pulmonary vein branches [Figure 18]. These are encircled with a vessel loop, elevated off of the PA emerging out of the pericardium, and divided using a stapler with a vascular cartridge. Next, the A3 PA branch is identified, encircled with a vessel loop, elevated, and divided using a stapling device [Figure 19]. The B3 bronchus emerges after dissecting the peribronchial N1 nodes that are between the PA and the bronchus [Figure 20]. The B3 bronchus is divided with a stapling device with a purple cartridge. Finally, the intersegmental fissure is identified as with the other segmentectomies and divided with a stapling device carrying a green cartridge [Figures 21 and 22]. 


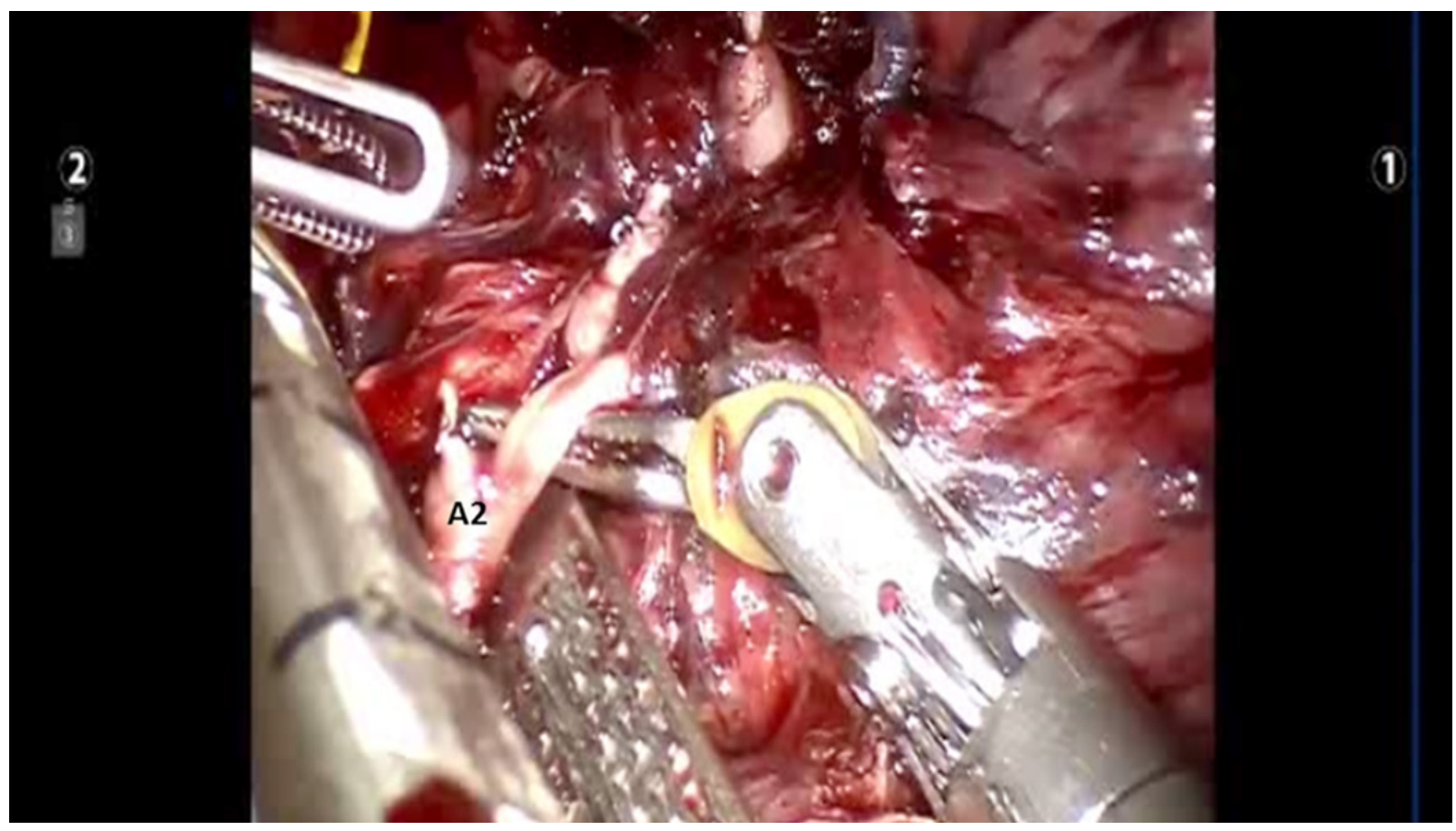

Figure 15. RS2 segmentectomy: Division of the V2 vein uncovers the A2 pulmonary artery branch

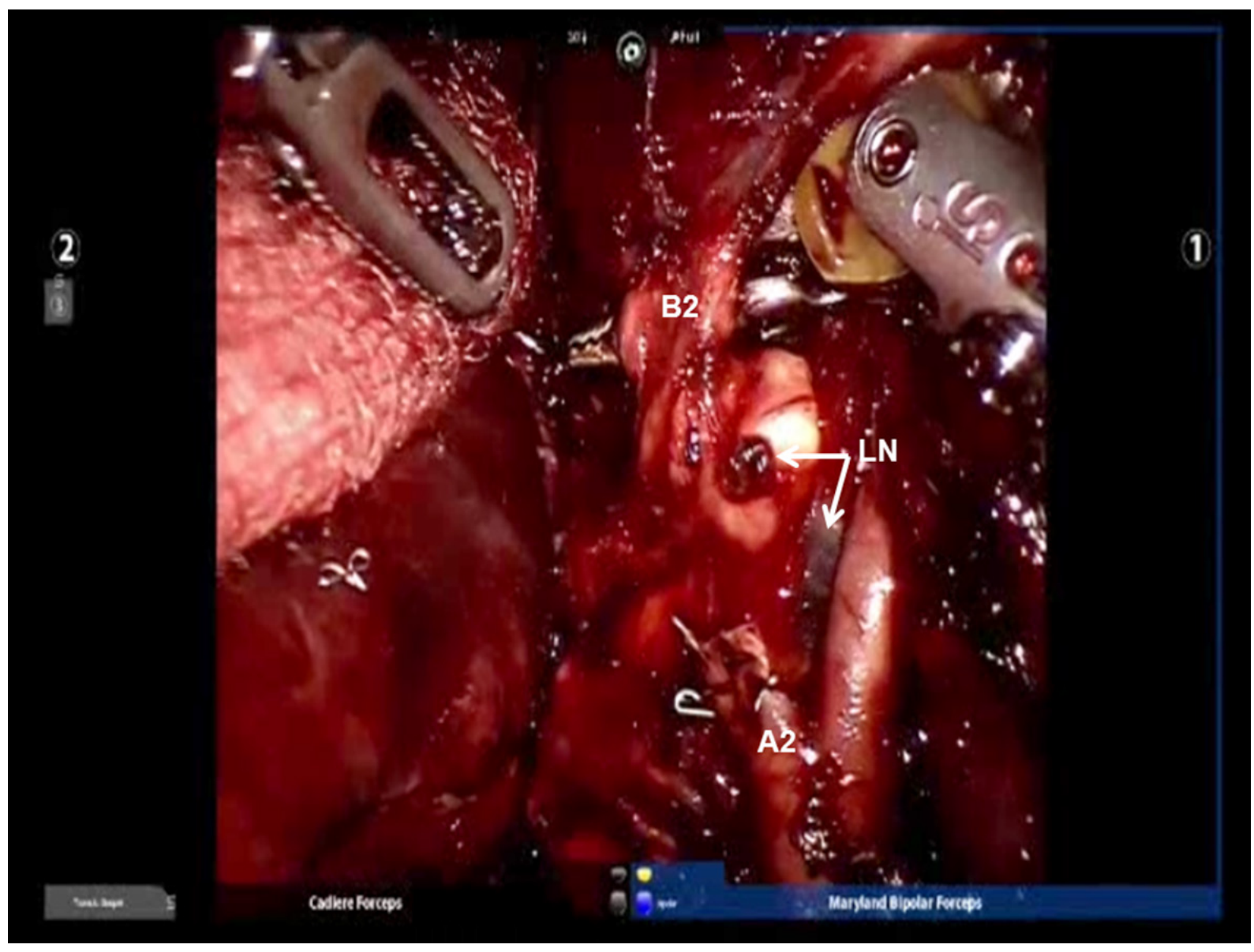

Figure 16. RS2 segmentectomy: The peribronchial N1 LN are removed completely, the B2 bronchus is encircled. The stapled stump of A2 branch of the pulmonary artery is seen. LN: lymph node 


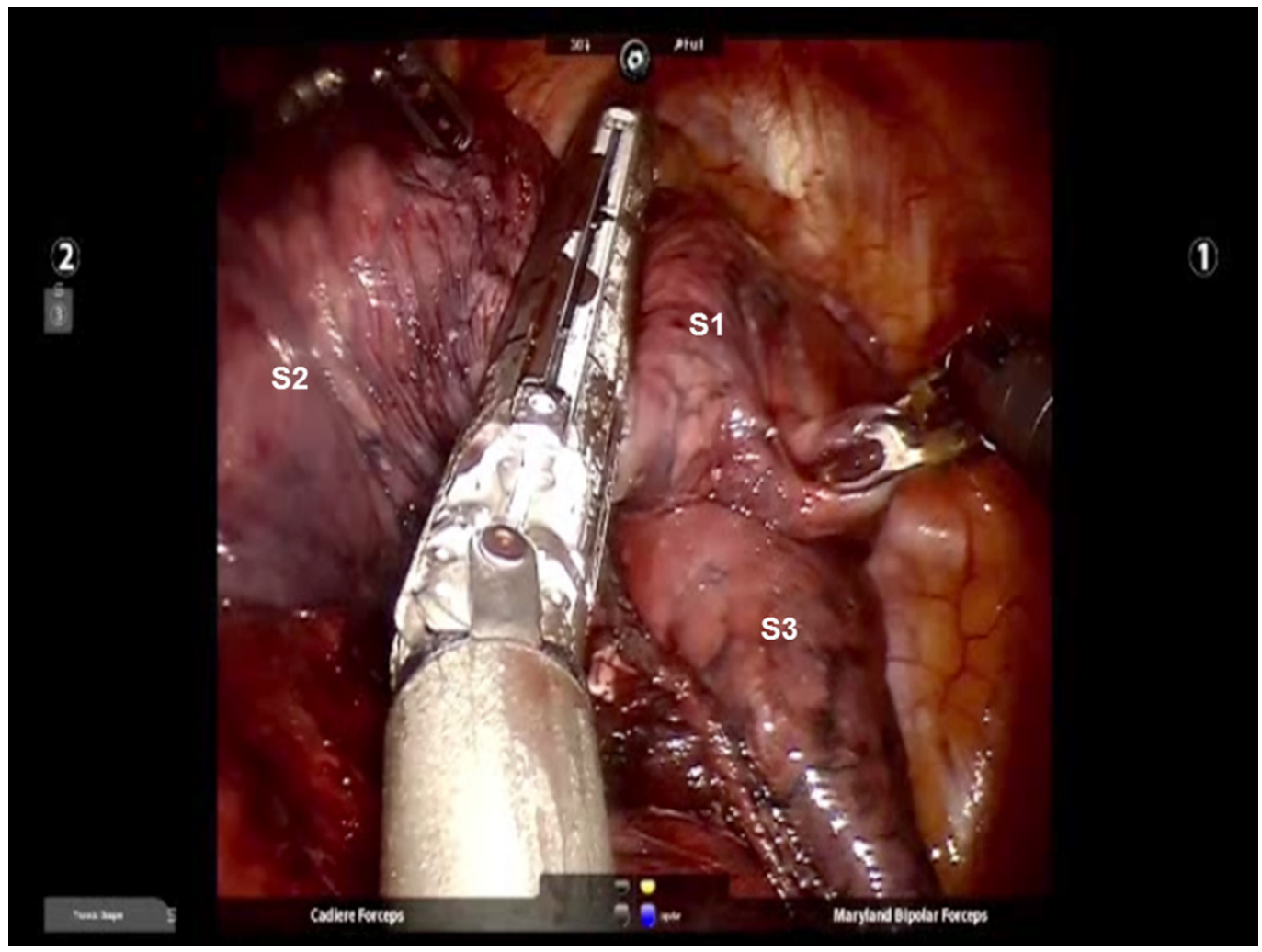

Figure 17. RS2 segmentectomy: The intersegmental fissure is identified by inflating the lung following the division of the B2 bronchus. The intersegmental fissure is divided with a stapling device. S1, S2, and S3 segments of the right upper lobe are seen

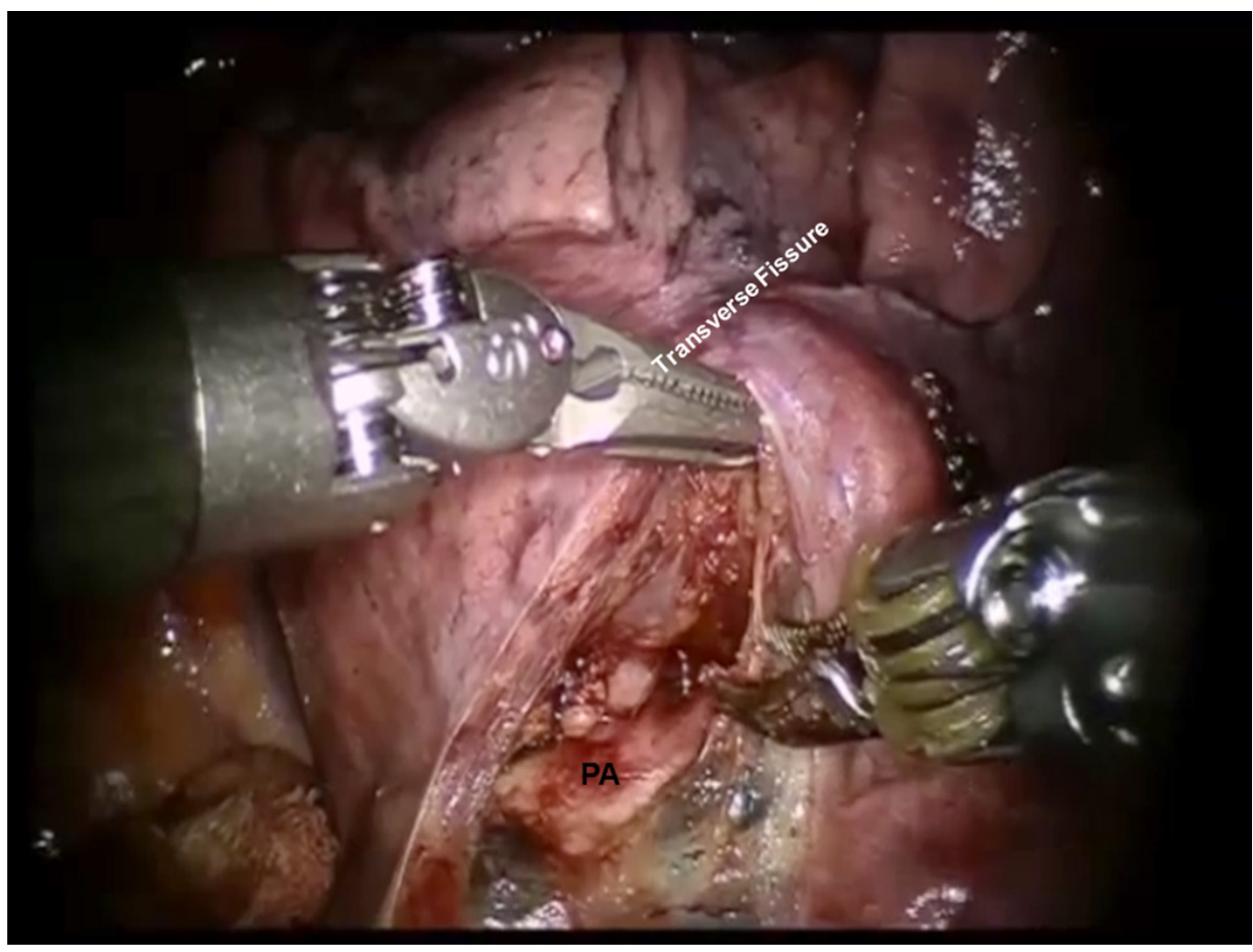

Figure 18. RS3 segmentectomy: The descending trunk of the PA is approached in the oblique fissure. Dissection is carried cephalad in the sub adventitial plane, which overlies the PA toward the main trunk heading toward the space between the pulmonary artery and superior pulmonary vein. PA: pulmonary artery 


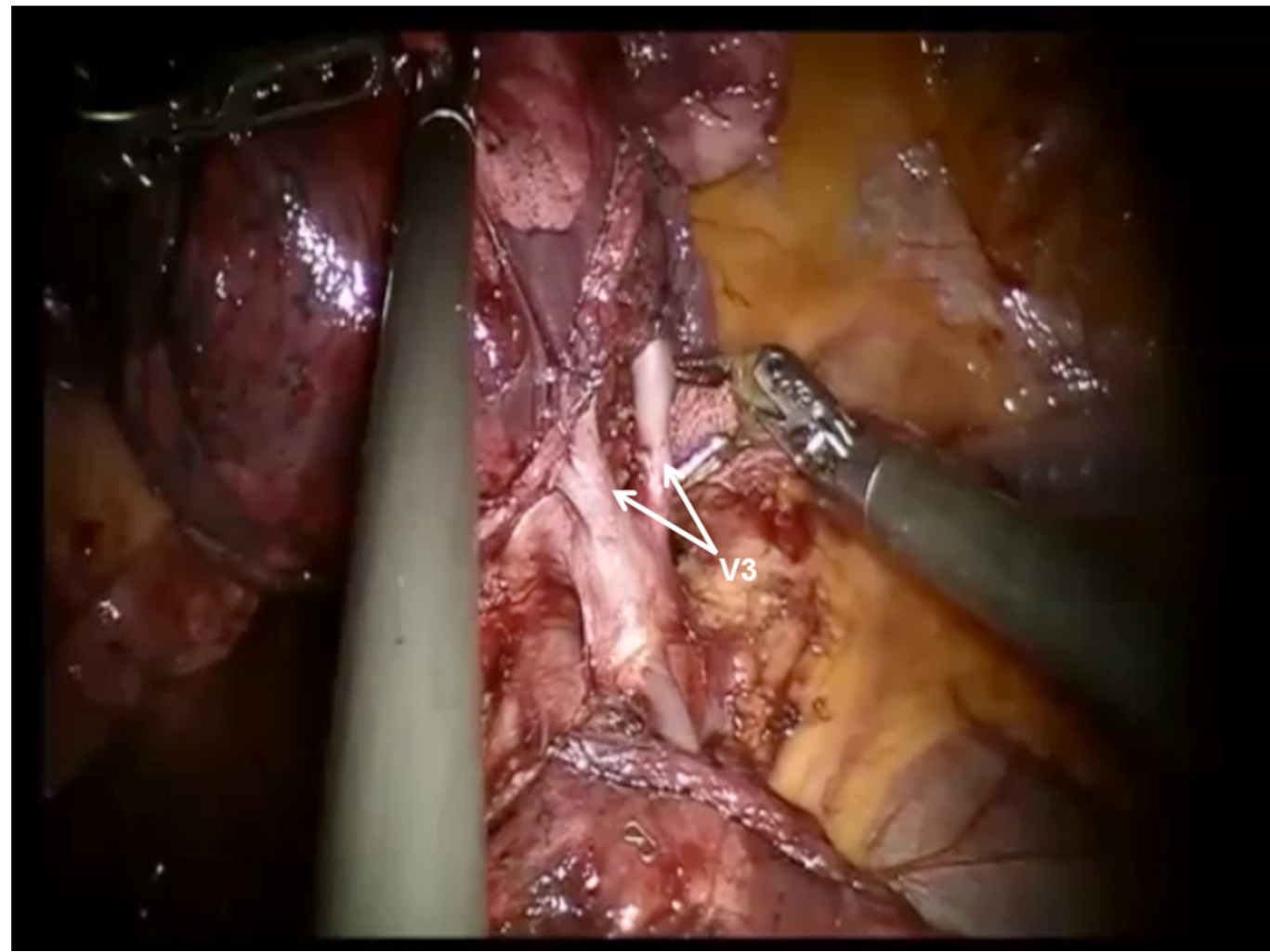

Figure 19. RS3 segmentectomy: Division of the transverse fissure exposes the V3 pulmonary vein branches

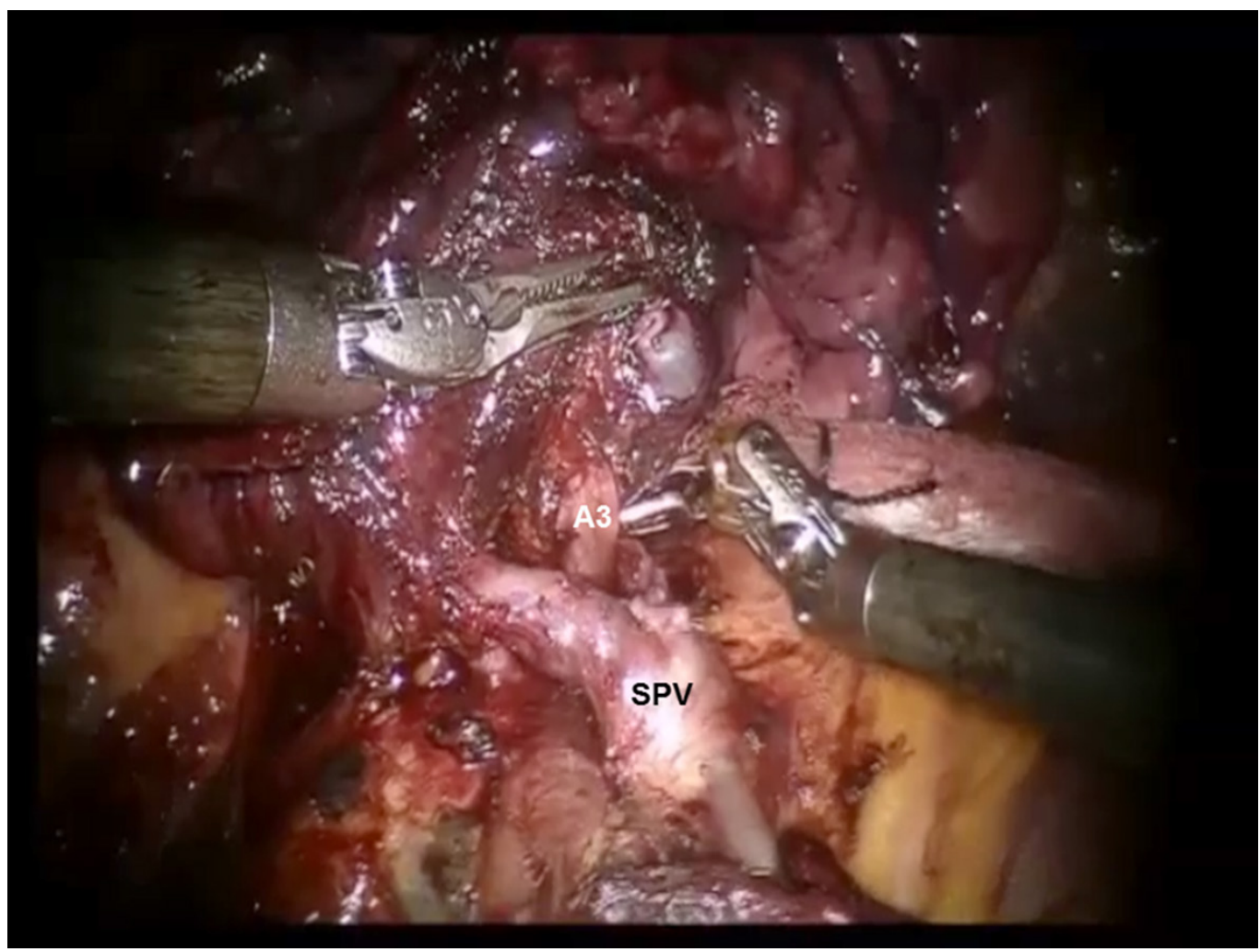

Figure 20. RS3 segmentectomy: Division of $\mathrm{V} 3$ exposes $A 3$ branch of the pulmonary artery. SPV: superior pulmonary vein 


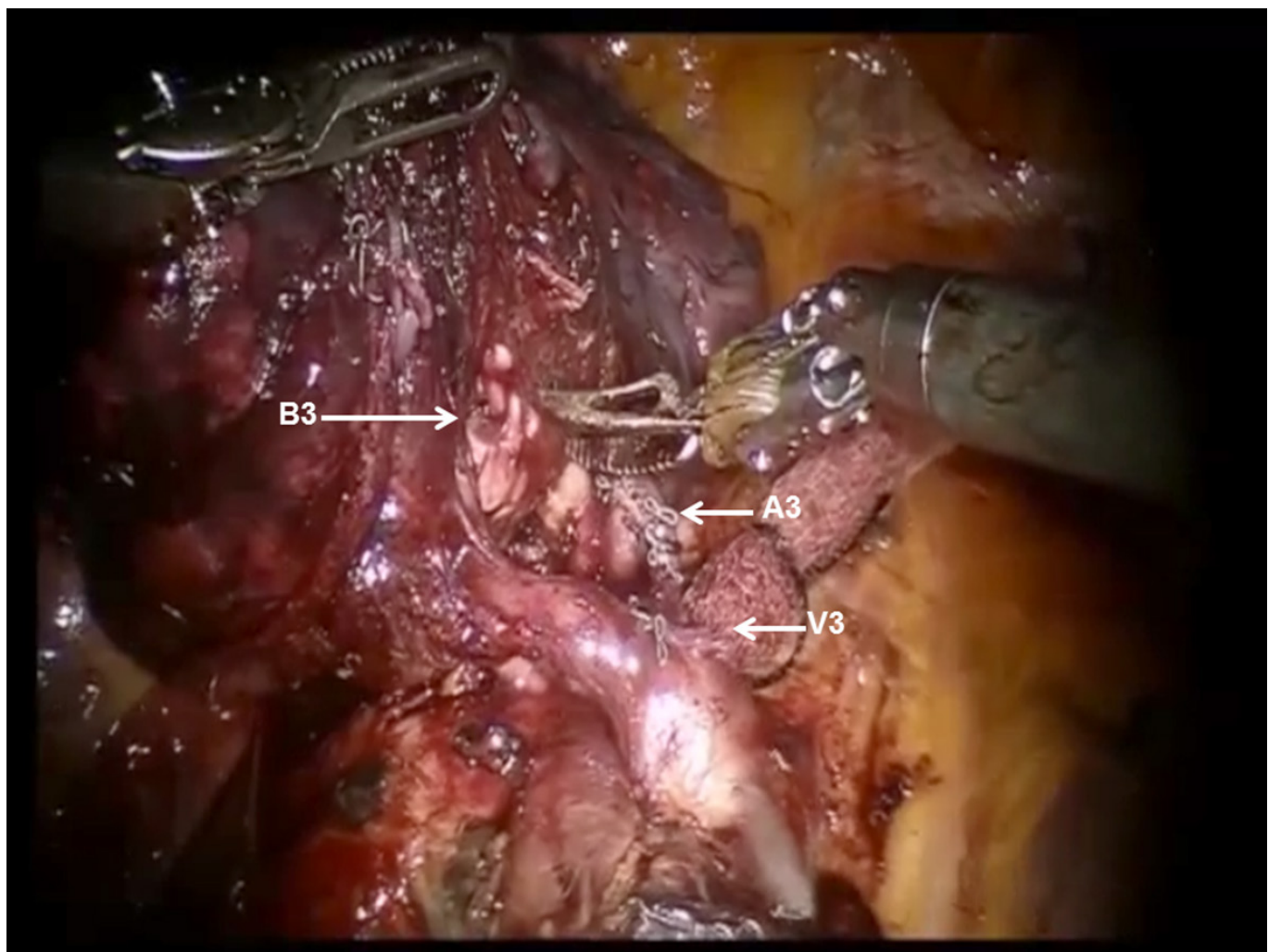

Figure 21. RS3 segmentectomy: The B3 bronchus emerges after dissecting the peribrochial $N 1$ nodes that are between the pulmonary artery branch (A3) and the bronchus. The stapled stump of $\mathrm{V} 3$ pulmonary vein branch is seen

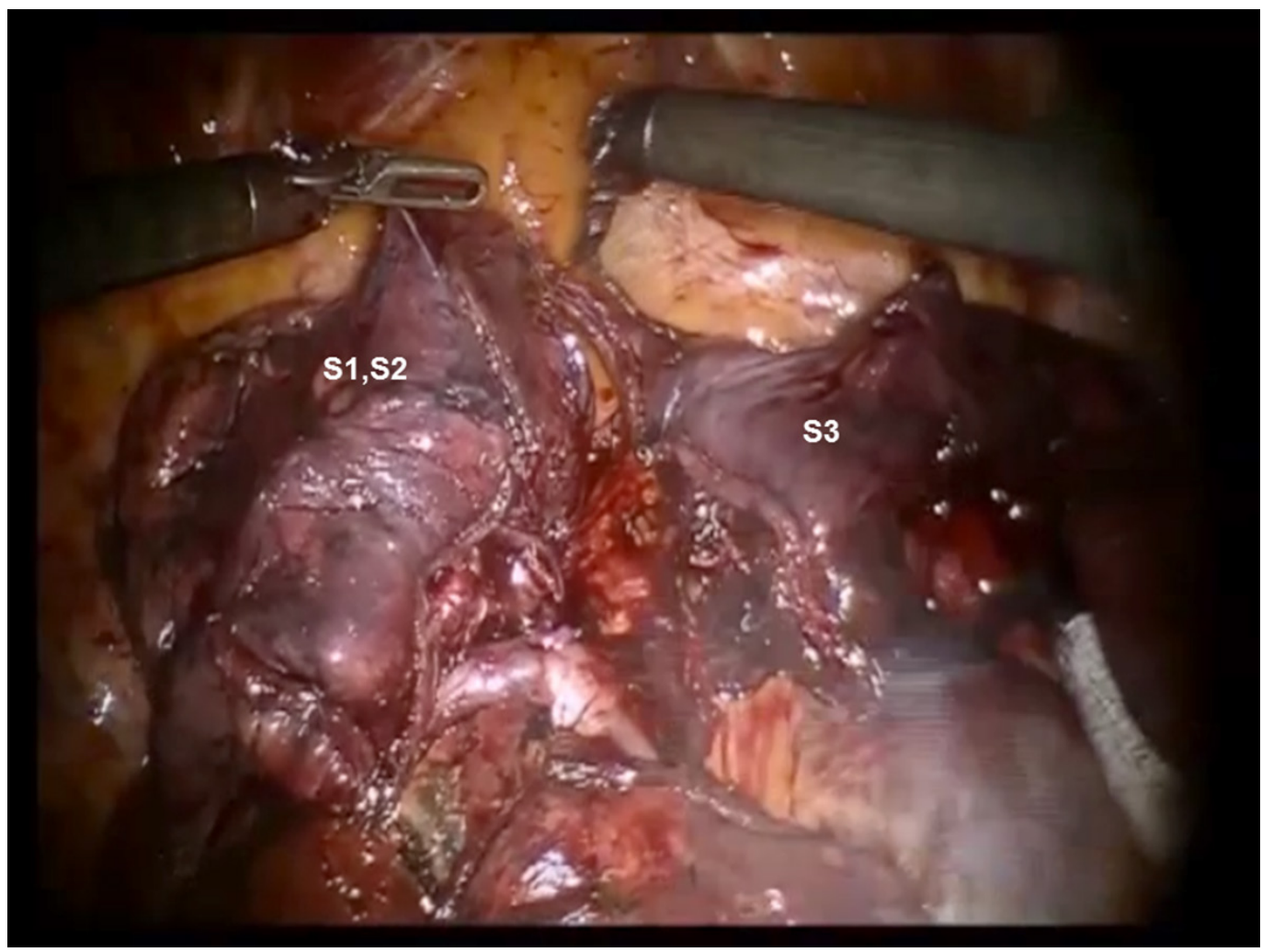

Figure 22. RS3 segmentectomy: Completed S3 segmentectomy. The separated S3 segment from S1 and S2 segments is seen 


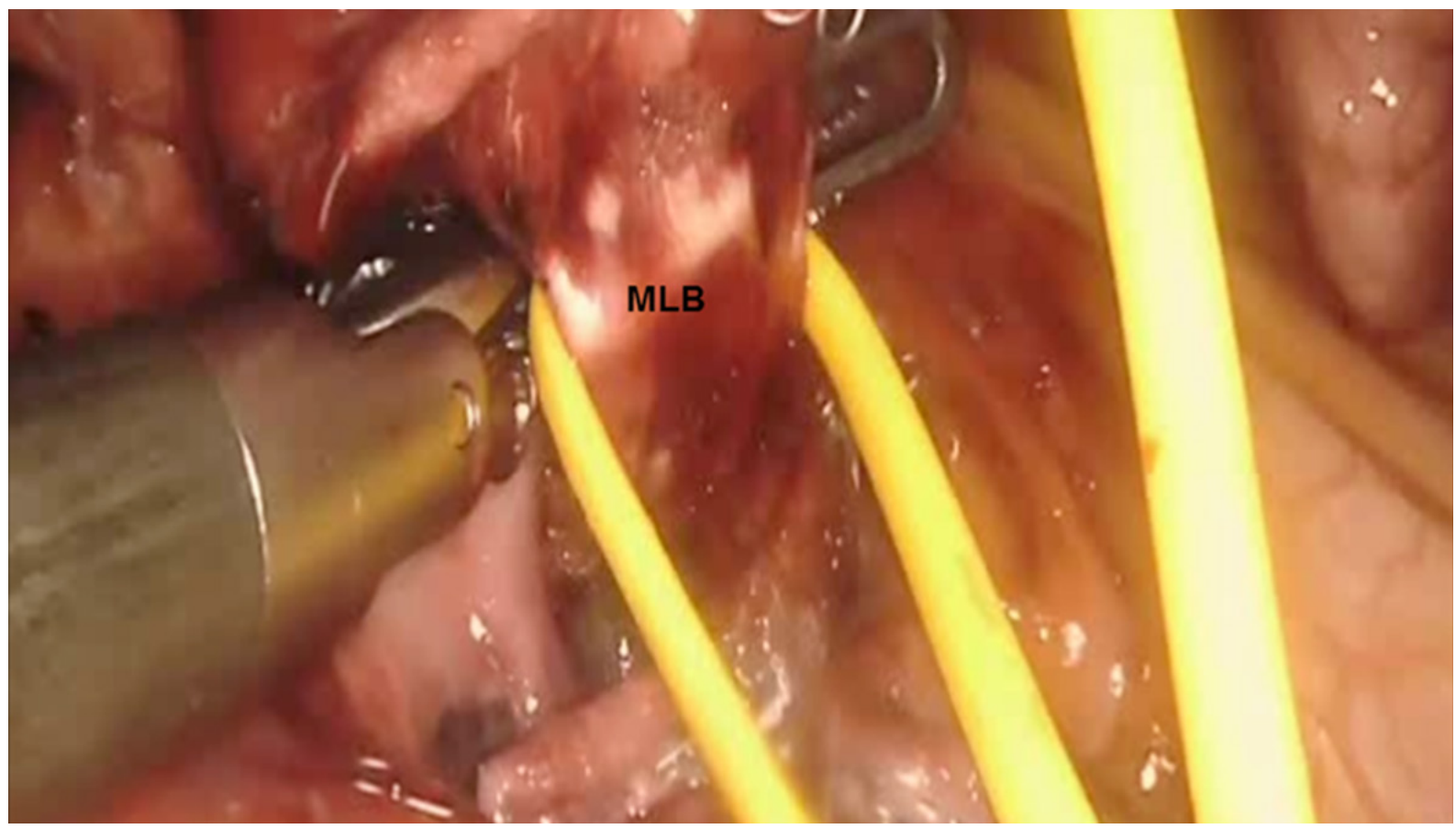

Figure 23. Right middle lobectomy ( $\mathrm{S} 4$ and S5): The MLB is identified. MLB: middle lobe bronchus

\section{Right middle lobe bi-segmentectomy (S4 and S5) = right middle lobectomy}

Although segmentectomy of S4 and S5 is technically possible, conventionally, a right middle lobectomy is performed.

Completion of the lymph node dissection opens the mediastinal space and facilitates the dissection of the artery and the bronchus. The lobectomy begins with retraction of the lung laterally and posteriorly with the most posterior robot arm. This helps expose the hilum.

The pleura posterior to the phrenic nerve is incised. The superior pulmonary vein is dissected. The bifurcation between the right upper and middle lobar veins is developed by dissecting it off the underlying PA. The right middle lobe vein is encircled and divided [Figure 23].

In our experience, the best way to enter the appropriate plane over the PA is to follow the anterior segmental branch to the lower lobe. This branch is usually very superficial and is not covered with nodal or parenchymal tissue. This branch can be followed superiorly to the main PA. This maneuver helps to elevate Station \#11 nodes off the PA and to identify the artery branch to the middle lobe. Next, the remainder of the fissure between the RML and RLL is divided in an anterior to posterior direction. At times, there is a vein branch to the middle lobe which drains into the inferior pulmonary vein. This is divided with the remainder of the anterior fissure.

Next, the middle lobe bronchus is identified [Figure 23]. It runs from left to right in the fissure. It is encircled and divided, taking care to avoid injuring the PA branches that are located directly behind it.

The middle lobe artery is encircled and divided with a vascular load. At times, the right middle lobe artery branches come off directly from the main PA instead of bifurcating from the common trunk of a single middle lobe artery. These are encircled and divided in the same fashion [Figure 24]. 


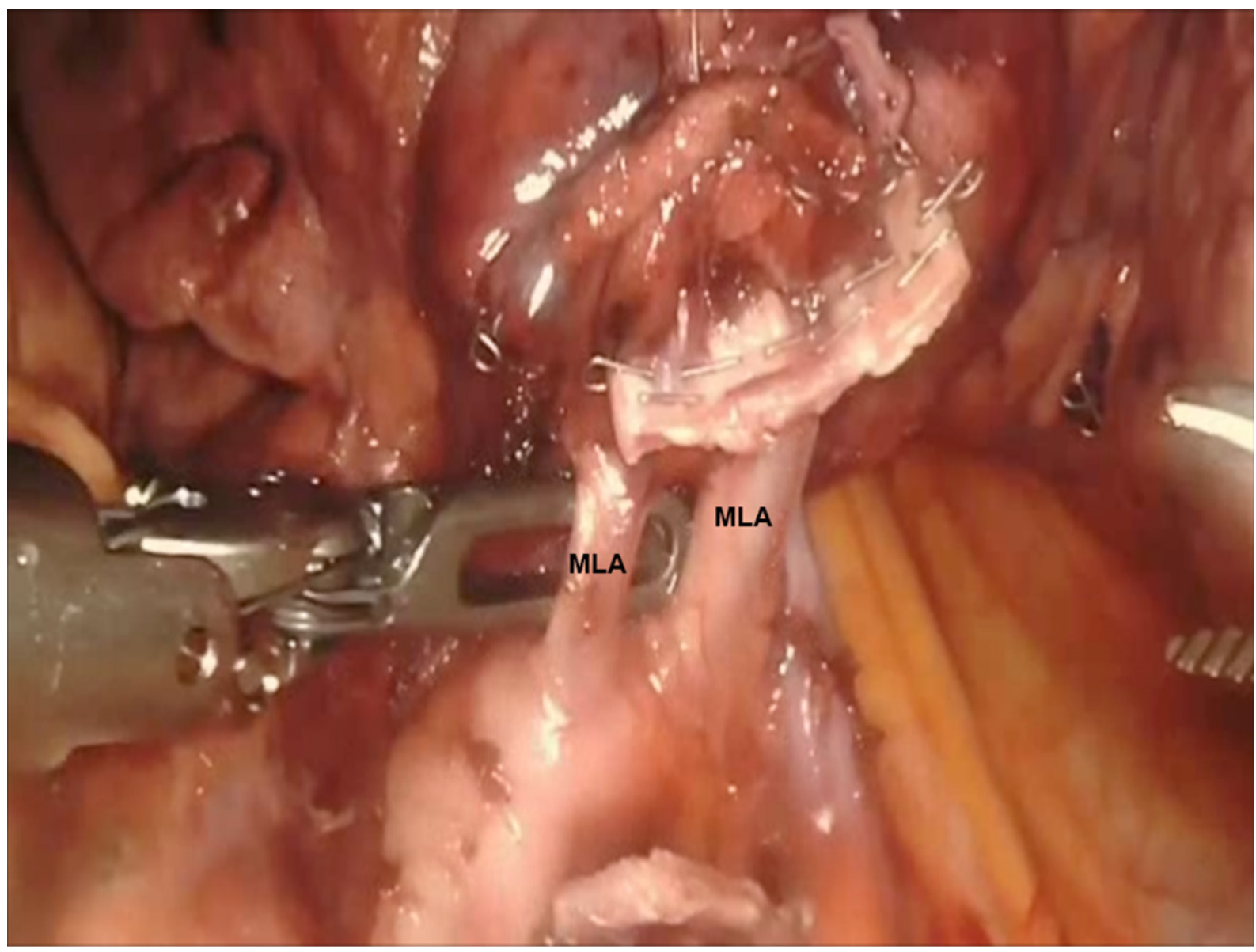

Figure 24. Right middle lobectomy (S4 and S5): The MLA are identified. MLA: middle lobe artery

Dissection of the fissure is then continued posteriorly until the main artery trunk and the superior segmental artery branch are identified. After identifying the main artery, the Cadiere Forceps in the left hand are used to go under the transverse fissure in a posterior to anterior direction heading for the divided superior pulmonary vein. A vessel loop is passed, and the fissure between the upper and middle lobes is divided using a stapler.

\section{Right lower lobe anatomic superior segmentectomy (S6)}

The docking, setup, and mediastinal nodal dissection is similar to right upper lobe anatomic segmentectomies.

The lung is retracted posteriorly and held in place with the robot arm. The bifurcation of the right superior and inferior pulmonary veins is dissected and delineated. The location of the right middle lobar vein should be positively identified to avoid inadvertent transection. The inferior pulmonary vein is encircled using the Cadiere Forceps.

The anterior branch of the lower lobe PA is most superficial and usually does not have overlying nodal tissue. This branch is identified and traced back to the main trunk of the PA. Next, the sub adventitial plane overlying the PA is developed and nodal tissue (Station \#11) is removed. Retraction is released and the lung is allowed to remain in its normal position, thereby facilitating visualization of the oblique fissure. The dissection is carried out posteriorly in the sub adventitial layer and the superior segmental branch of the 


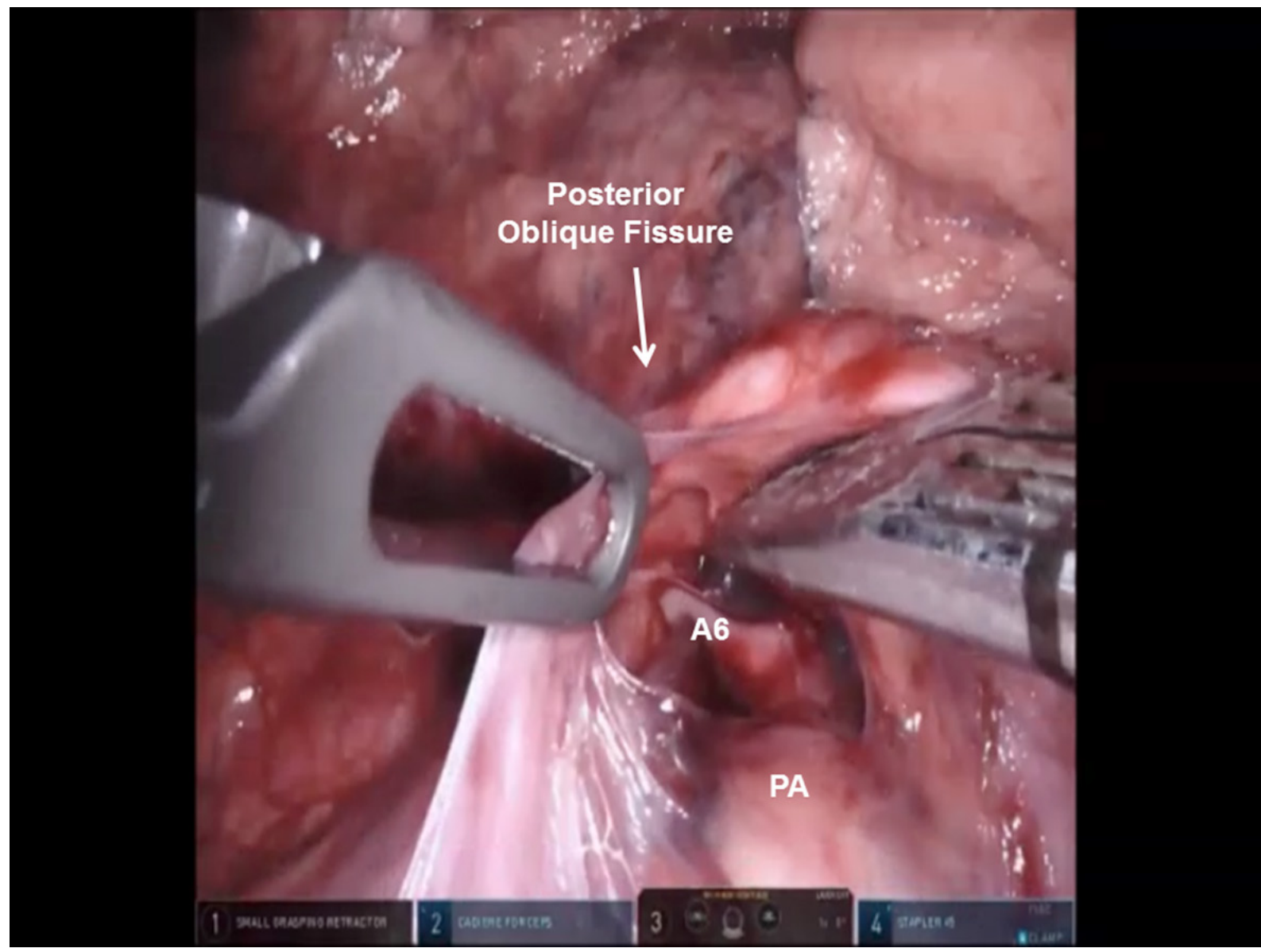

Figure 25. RS6 segmentectomy: The posterior oblique fissure is divided and the A6 branch of the descending PA is identified. PA: pulmonary artery

lower lobe PA is identified. The major fissure is then divided from an anterior to posterior direction using a stapler, which is introduced from the anterior port [Figure 25].

The A6 branch of the descending PA is identified, cleared of nodal tissue, encircled, and divided with a vascular stapler [Figure 26]. The nodes overlying the right lower lobe bronchus are swept toward the specimen, and the B6 bronchus is identified, encircled, and divided [Figure 27]. The intersegmental fissure between the $\mathrm{S} 6$ and the basal segments of the lower lobe is identified, as outlined above, and divided using a stapling device [Figure 28].

\section{Right lower lobe anatomic basal segmentectomy (S7-S10)}

Most surgeons would perform a formal lower lobectomy instead of a lower lobe basal segmentectomy. However, the approach to this segmentectomy is similar to superior segmentectomy (S6). Following the complete mediastinal nodal dissection and removal of Station \#9, \#7, \#4L, \#5, \#10, and \#11 nodes, the inferior pulmonary vein is encircled with a vessel loop and elevated. The superior segmental vein is identified, thereby allowing for identification of the basal branch of the inferior pulmonary vein. The basal vein is then divided with a stapling device with a white cartridge. Next, the PA is isolated in the fissure, as described above. The right lower lobe PA is identified, encircled, and elevated with a vessel loop. The basal branch of the right PA is divided with a vascular stapler. Following the division of the basal PA, the bronchus to the basal segment (B7-10) is encircled and divided with a stapler carrying a blue cartridge. Finally, the intersegmental fissure is identified and divided using a stapler with a green cartridge. 


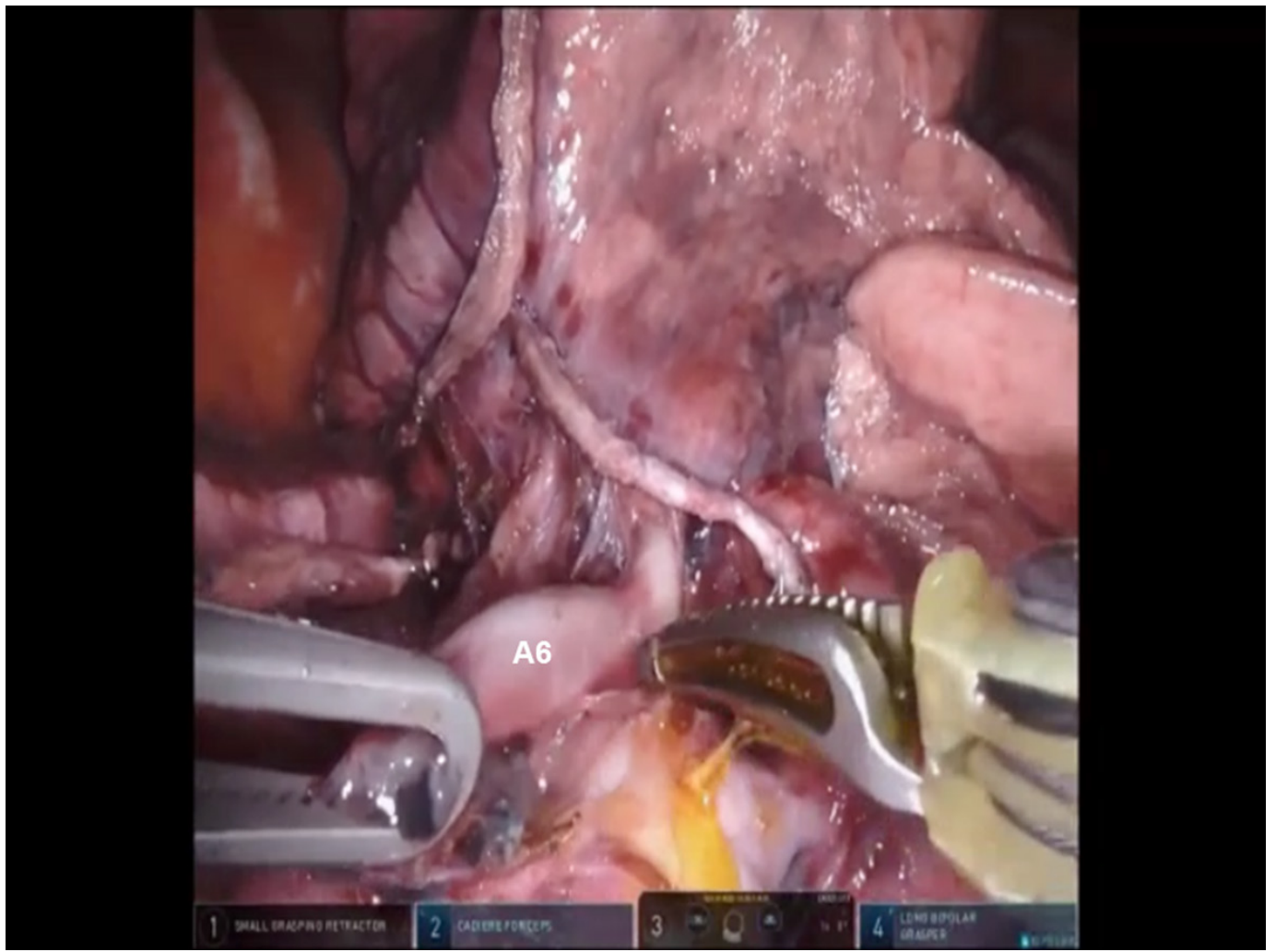

Figure 26. RS6 segmentectomy: The A6 branch of the descending pulmonary artery is skeletonized and divided

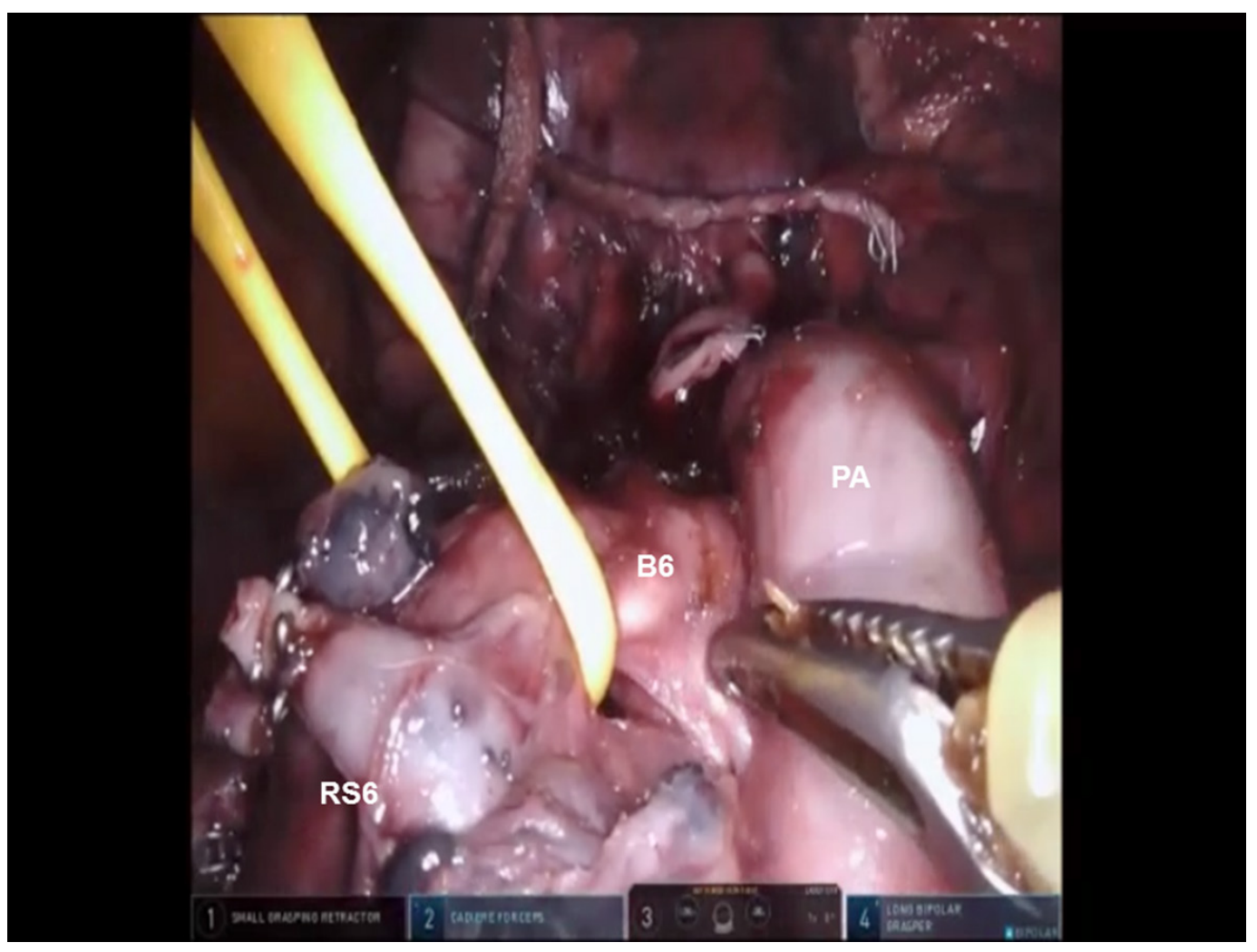

Figure 27. RS6 segmentectomy: B6 bronchus is identified and encircled prior to division. PA: pulmonary artery; RS: superior segment of right lower lobe 


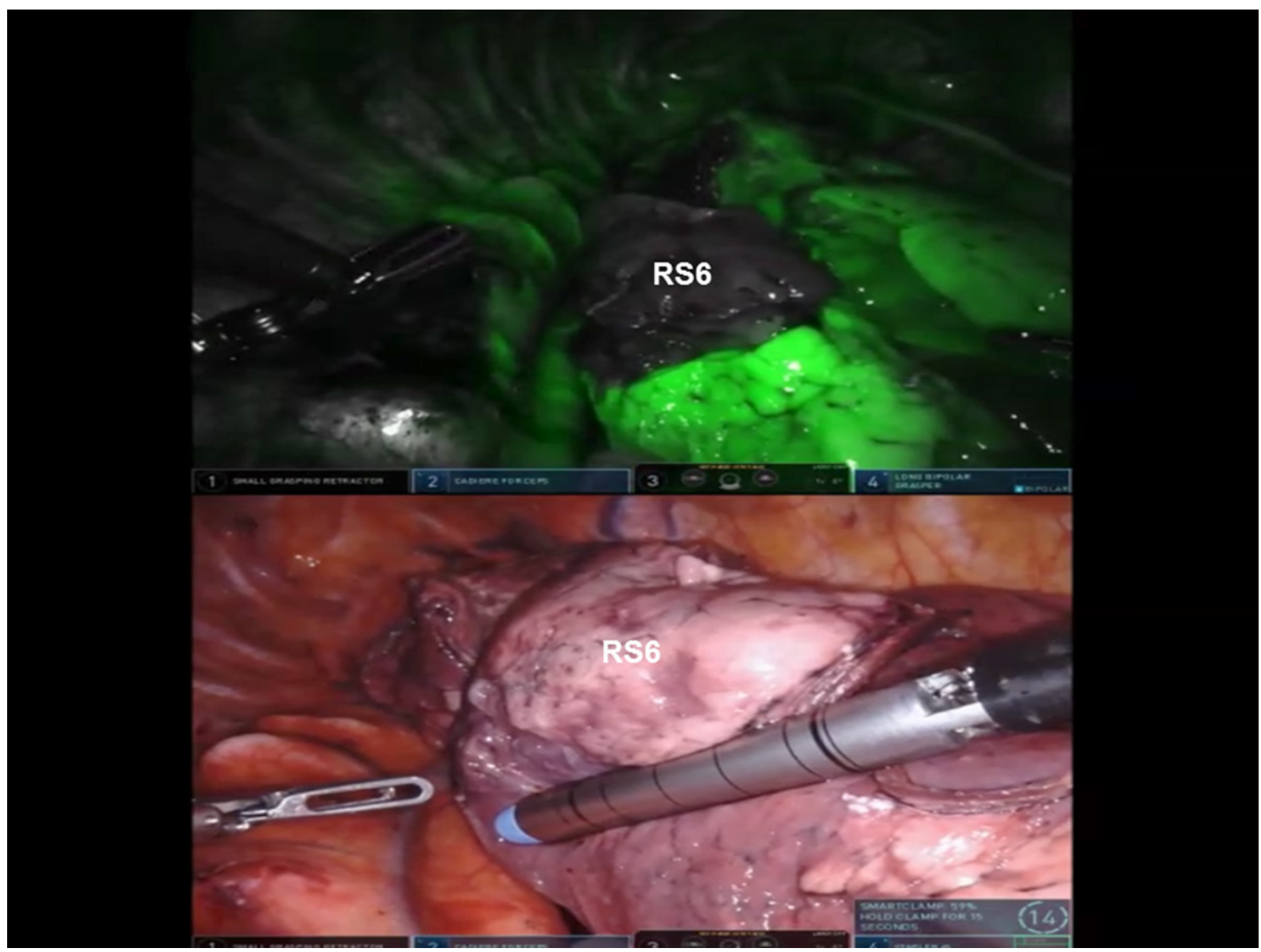

Figure 28. RS6 segmentectomy: The intersegmental fissure between the RS6 (superior segment right lower lobe) and the basal segments of the lower lobe is identified and divided

\section{CONCLUSION}

The surgical robot allows for precise dissection of the segmental bronchopulmonary structures, while minimizing trauma to surrounding tissues, and it allows for thorough and complete dissection of the mediastinal nodes. Robotic segmentectomy should be considered when planning a lung sparing operation in patients with smaller tumors or for physiologic considerations. The long-term results of robotic anatomic segmentectomy from our Institution were reported by Nguyen et al. ${ }^{[19]}$ Mean operative time was 134 min (range 70-227 min). Median length of stay was seven days (range 2-31 days). There were no conversions to robotic lobectomy. Two of $61(3 \%)$ patients were converted to thoracotomy due to tumor location. Complications were minor (29\%) with the most common being atrial fibrillation. There was no mortality. In patients with pathologic stage I NSCLC undergoing robotic anatomic segmentectomy, the lung cancerspecific five-year actuarial survival was $73 \%{ }^{[19]}$.

The advantages of the use of robotic technology should be viewed in the perspective of increased cost and a steep learning curve for this complex procedure.

\section{DECLARATIONS}

\section{Authors' contributions}

Contributed equally to the performance of the surgeries, collection of data and writing the manuscript: Gharagozloo F, Meyer M 


\section{Availability of data and materials}

Not applicable.

\section{Financial support and sponsorship}

None.

\section{Conflicts of interest}

All authors declared that there are no conflicts of interest.

\section{Ethical approval and consent to participate}

Not applicable.

\section{Consent for publication}

Not applicable.

\section{Copyright}

(c) The Author(s) 2020.

\section{REFERENCES}

1. Fontana RS, Sanderson DR, Woolner LB, Taylor WF, Miller WE, et al. Lung cancer screening: the Mayo Program. J Occup Med $1986 ; 28: 746-50$

2. Marcus P, Bergstralh E, Fagerstrom R, Williams DE, Fontana R, et al. Lung cancer mortality in the Mayo Lung Project: impact of extended follow up. J Natl Cancer Inst 2000;92:1308-16.

3. Narsule CK, Ebright MI, Fernando HC. Sublobar versus lobar resection: current status. Cancer J 2011;17:23-7.

4. Miller DL, Rowland CM, Deschamps C, Allen MS, Trastek VF, et al. Surgical treatment of non-small cell lung cancer $1 \mathrm{~cm}$ or less in diameter. Ann Thorac Surg 73:1545-50.

5. Okada M, Yoshikawa K, Hatta T, Tsubota N. Is segmentectomy with lymph node assessment an alternative to lobectomy for non-small cell lung cancer of $2 \mathrm{~cm}$ or smaller? Ann Thorac Surg 2001;71:956-61.

6. Smith CB, Swanson SJ, Mhango G, Wisnivesky JP. Survival after segmentectomy and wedge resection in stage I non-small cell lung cancer. J Thorac Oncol 2013;8:73-8.

7. Sienel W, Dango S, Kirschbaum A, Cucuruz B, Stremmel WHC, et al. Sublobar resections in stage IA non-small cell lung cancer: segmentectomies result in significantly better cancer-related survival than wedge resection. Eur J Cardiothorac Surg 2008;33:728-34.

8. Altorki NK, Kamel MK, Narula N, Ghaly G, Nasar A, et al. Anatomical segmentectomy and wedge resections are associated with comparable outcomes for patients with small cT1N0 non-small cell lung cancer. J Thorac Oncol 2016;11:1984-92.

9. Kilic A, Schuchert MJ, Pettiford BL, Pennathur A, Landreneau JR, et al. Anatomic segmentectomy for stage I non-small cell lung cancer in the elderly. Ann Thorac Surg 2009;83:1662-6.

10. El-Sherif A, Gooding WE, Santos R, Pettiford B, Ferson P, et al. Outcomes of sublobar resection versus lobectomy for stage I non-small cell lung cancer: a 13-year analysis. Ann Thorac Surg 2006; 82:408-16.

11. Schuchert MJ, Pettiford BL, Keeley S, D’Amato TA, Kilic A, et al. Anatomic segmentectomy in the treatment of stage I non-small cell lung cancer. Ann Thorac Surg 2007;84:926-33.

12. Cao C, Chandrakumar D, Gupta S, Yan TD, Tian DH. Could less be more?-A systematic review and meta-analysis of sublobar resections versus lobectomy for non-small cell lung cancer according to patient selection. Lung Cancer 2015;89:121-32.

13. Okami J, Ito Y, Higasgyyama M, Nakayama T, Tokunaga T, et al. Sublobar resection provides an equivalent survival after lobectomy in elderly patients with early lung cancer. Ann Thorac Surg 2010;90:1651-6.

14. Dylewski MR, Ohaeto A, Pereira J. Pulmonary resection using a total endoscopic robotic video-assisted approach. Semin Thorac Cardiovasc Surg 2011;23:36-42.

15. Pardolesi A, Park B, Petrella F, Borri A, Gasparri R, et al. Robotic anatomic segmentectomy of the lung: technical aspects and initial results. Ann Thorac Surg 2012;94:929-34.

16. Cerfolio RJ, Bryant AS, Skylizard L, Minnich DJ. Initial consecutive experience of completely portal robotic pulmonary resection with 4 arms. J Thorac Cardiovasc Surg 2011;142;740-6.

17. Cerfolio RJ, Watson C, Minnich DJ, Callaway S, Wei B. One hundred planned robotic segmentectomies: early results, technical details, and preferred port placement. Ann Thorac Surg 2016;101:1089-95.

18. Demir A, Ayalp K, Ozkan B, Kaba E, Toker A. Robotic and video-assisted thoracic surgery lung segmentectomy for malignant and benign lesions. Interact Cardiovasc Thorac Surg 2015;20:304-9.

19. Nguyen D, Gharagozloo F, Tempesta B, Meyer M, Gruessner A. Long-term results of robotic anatomical segmentectomy for early-stage non-small-cell lung cancer. Eur J Cardiothorac Surg 2019;55:427-33. 